\title{
Stepwise approach to oocyte depletion in Sry mutated XY female mice
}

Akihiko Sakashita ${ }^{1}$, Takuya Wakai $^{1 \dagger}$, Yukiko Kawabata ${ }^{1}$, Chiaki Nishimura ${ }^{1}$, Yusuke Sotomaru ${ }^{2}$, Tomohiro Kono ${ }^{1 \#}$

1. Department of Bioscience, Tokyo University of Agriculture, Setagaya, Tokyo, Japan

2. Natural Science Centre for Basic Research and Development, Hiroshima University, Hiroshima, Japan

\#Corresponding author: Department of BioScience, Tokyo University of Agriculture, 1-1-1 Sakuragaoka, Setagaya-ku, Tokyo 156-8502, Japan.

E-mail: tomohiro@nodai.ac.jp

${ }^{\dagger}$ Present address: Graduate School of Environment and Life Science, Okayama University, 3-1-1, Tsushimanaka, Kitaku, Okayama 700-8530, Japan.

Keywords: Sex-reversed mice, Infertility of XY female, XY PGCs/oocytes, Transcriptome analysis, Germ cell depletion, Wnt signaling, Cell death program. 


\begin{abstract}
Little is known regarding the mechanisms underlying infertility in male-to-female sex-reversed females. To gain a better understanding of germ cell dysfunction in this condition, we produced $X Y^{\text {sry-}}$-females via the CRISPR/Cas9 system in C57BL/6 inbred strain mice. Mutant mice showed severe attrition of germ cells during foetal development, resulting in depletion of ovarian germ cells before sexual maturation. Comprehensive transcriptome analysis of embryonic day 13.5 primordial germ cells (PGCs) and postnatal day 1 oocytes demonstrated that $X Y^{\text {sry-}}$-PGCs had already deviated from the developmental process at the

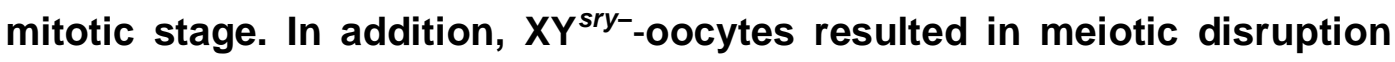
and developmental failure, and the genes related to these processes were significantly changed. This grievous disruption, which caused germ cell deterioration in $\mathrm{XY}^{\text {sry-}}$-females, was shown to proceed from the germ cells themselves. These results provide novel insight into the germ cell depletion of sex-reversed mice as well as into disorders of sex differentiation in human, such as 'Swyer syndrome', wherein patients present as typical females albeit with an XY karyotype and infertile.
\end{abstract}




\section{Introduction}

Sex determining region $\mathrm{Y}($ Sry), is widely conserved in mammals and acts as a determinant in the 'bipotential' gonads present early in development for male sex-inducing testis formation (Kashimada and Koopman, 2010; Tanaka and Nishinakamura, 2014). In contrast, XX female embryos automatically develop into females, forming ovaries chiefly of oocytes and follicle cells (Suzuki et al., 2015). Failure of the sex differentiation process causes various types of genetic diseases termed disorders of sex differentiation in human (Jiang et al., 2016; Tam et al., 2016; Zhong and Layman, 2012). In mice, Sry expression occurs in GATA binding protein 4- and Wilms tumor 1 homolog-expressing gonadal somatic cells of the genital ridges at embryonic day (E) 11.0-12.0 (Kashimada and Koopman, 2010; Miyamoto et al., 2008). This expression directly supports gonadal cell precursors such as Sertoli cells that the fate of bipotential gonads is specified by $E 13.5$, resulting in their subsequent differentiation into either ovaries or testes. In particular, Sry is expression triggers the activation of the male sex differentiation program and induces the expression of SRY-related high-mobility group box 9 (Sox9), and fibroblast growth factor 9 , which leads to male gonad differentiation accompanied by formation of pre-spermatogonia and Sertoli cells (Kashimada and Koopman, 2010; Tanaka and Nishinakamura, 2014).

Notably, Sry mutations are well-known to result in male-to-female sex-reversed individuals. Such sex-reversed mice (XY-females) were first produced from chimeric mice via embryonic stem (ES) cells that carried a mutation at the testis-determining region of the $\mathrm{Y}$ chromosome ( $T d y$ ) by using a retroviral vector (Gubbay et al., 1990; Gubbay et al., 1992; Lovell-Badge and Robertson, 1990). More recently, a TALEN-mediated gene editing procedure has been used to target specific $Y$ chromosome genes, resulting in the production of anatomical sex-reversed $X Y$ females were produced by deletion of the HMG domain of Sry (Kato et al., 2013; Wang et al., 2013). These animals were found to be infertile: however detailed analysis for infertility was not performed because the necessary number of $X Y$-females was unavailable. $X Y$ female mice have also been produced by the deletion of autosomal Sry-related 
genes. For example, the deletion of Sox9 in the gonadal somatic cells of male embryos resulted in their development into sex-reversed $X Y$ females (Lavery et al., 2011). In addition, the alteration of histone epigenetic signatures through deletion of the JmjC-containing H3K9 demethylase Jmjd1a, which positively regulates Sry expression, also resulted in male-to-female sex-reversed mice (Kuroki et al., 2013). Furthermore, it is known that male-to-female sex-reversed mice are produced when C57BL/6 mice are mated with certain strains of Mus musculus, such as B6-Y $Y^{A K R}, B 6-Y^{P O S}$, or B6-Y $Y^{T I R}$ (Correa et al., 2012; Coward et al., 1994; Eicher et al., 1982; Lee and Taketo, 1994; Washburn et al., 2001). Specifically, these $X Y$ female mice exhibit an anatomically female phenotype with ovary formation; however, they are generally infertile, exhibiting extensive loss of germ cells in the ovary except in a few cases comprising hybrid strains (Kato et al., 2013; Lovell-Badge and Robertson, 1990; Mahadevaiah et al., 1993; Park and Taketo, 2003; Taketo, 2015; Vanderhyden et al., 1997; Vernet et al., 2014b; Wang et al., 2013). Overall, it is evident that $X Y$ females form well-constructed foetal gonads consisting of sites of cyst formation, pregranulosa cells, and oogonia. However, if oocytes from XY females are ovulated and fertilized they cease development at a very early stage (Vernet et al., 2014b; Villemure et al., 2007; Wang et al., 2013; Wong et al., 2000).

In comparison, unlike in human, XO mice are fertile (Cloutier et al., 2015; Probst et al., 2008), which indicates that genes located on the $Y$ chromosome exert a pivotal effect on germ cell fate in $X Y$ females. Using a genetic model of $\mathrm{XO}$ mice carrying genes located on the short arm of the $Y$ chromosome, the function of the Y-associated genes has been investigated (Vernet et al., 2014b). For example, Zfy2 are shown to be essential for promoting Meiosis II (Vernet et al., 2011; Vernet et al., 2014a), whereas the testis determinant factor Sry and the spermatogonial proliferation factor eukaryotic translation initiation factor 2 , subunit 3, structural gene Y-linked (Eif2s3y) were found to constitute minimum factors for producing live offspring from XO males, by ROSI (Yamauchi et al., 2014). In addition, deletion of Eif2s3y resulted in infertility because of a defect in spermatogenesis (Matsubara et al., 2015), whereas mutations of ubiquitously transcribed tetratricopeptide repeat gene showed no effect (Wang et al., 2013). 
Furthermore, every sex-reversed mouse tested was infertile; therefore, the specific molecular mechanisms underlying the generation of germ cell developmental defects in XY-female mice remained to be elucidated.

To gain a better understanding of the dysfunction of germ cells, developmental defects and infertility in male-to-female sex-reversed mice, we

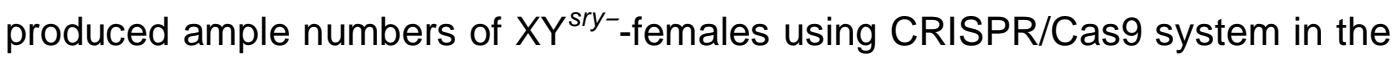
inbred C57BL/6 mouse strain, which is necessary to minimize experimental variation. Focusing on germ cell proliferation, attrition and functionality, comprehensive comparative transcriptomic analysis was conducted at E13.5, postnatal day (P) 1 and juvenile (4-weeks) stages. From these data, the gene sets specific for sex-reversed $X Y^{\text {sry- }}$-germ cells were identified and subjected to further bioinformatics analysis. The present study demonstrated that $X Y^{\text {sry-}}$-primordial germ cells (PGCs) of sex-reversed mice with Sry-mutation already had deviated from the developmental process at the meiotic stage. Furthermore, this disruption, which caused germ cell deterioration in $X Y^{\text {sry-}}{ }^{-}$females, appeared to proceed from the germ cells, themselves.

\section{Results}

\section{Phenotype of Sry targeted mice}

In total, we generated and used 65 sex-reversed $X Y^{\text {sry-}}{ }^{\text {-female mice for germ }}$ cell analysis (Fig S1A), in which part of the Sry sequence adjacent to the transcription start site was deleted using the CRISPR/Cas9 system. The generated mice were anatomically normal females, although slight aplasia was detected in the reproductive organs (Fig. 1A). Sequence analysis detected either $1 \mathrm{bp}-14$ bp deletions or large insersions (500 bp<) in the Sry gene (Fig. $\mathrm{S} 1 \mathrm{~B})$, which led to framesifts of the gene and resulted in the loss of function in all cases. Of the mutations detected in the $X Y^{\text {sry-}}{ }^{-}$-female mice, 7 bp deletions $(n=23.4 \%)$ most frequently occurred (Fig. S1). All mutations detected in $X Y^{\text {sry-}}{ }^{-}$-females led to translational frameshifts in SRY and its functional failure. Mating tests of $14 X Y^{\text {sry-}}{ }^{-}$females (8-10 weeks old) with normal $X Y$ males revealed the infertility of the sex-reversed mice (Fig.1B). To determine the cause of infertility, the numbers of PGCs and oocytes were examined. Notably, a 
significant decrease in PGC number was already found at E13.5 in the

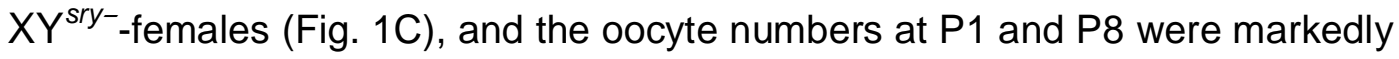
decreased to $1 / 5$ and $1 / 6$ compared with those of controls, respectively. Immunofluorescence staining of DDX4, a germ cell maker, indicated that fewer positive cells were apparent in $X Y$ females. In particular, the primary follicle oocytes were lost by the P8 stage in $X Y^{\text {sry-}}{ }^{-}$-females (Fig. 1D). The decrease of PGCs in the $X \mathrm{Y}^{\text {sry-}}{ }^{\text {-}}$-female ovary progressed quickly after mid gestation, finally resulting in ovarian atrophy accompanied with oocyte depletion by 6 weeks of age (Fig. 1E). These results revealed that the infertility of sex-reversed $X Y^{\text {sry-}}$-female mice was caused by complete depletion of the germ cells.

\section{Transcriptome analysis of $X Y^{\text {sry-}}$-germ cells}

To understand the mechanisms underlying the depletion of germ cells in $X Y^{\text {sry-}}{ }^{-}$females, we conducted transcriptome analysis followed by bioinformatics analysis. The RNA-Seq datasets provided accurate gene expression profiles: a summary of the gene expression profiles is shown in Table S2 and S3. Hierarchical cluster analysis revealed that the gene expression profiles segregated into two groups representing E13.5 PGCs and P1 oocytes and that both profiles of the $X \mathrm{X}^{\text {sry-}}$-female germ cells could be clearly distinguished from those of $X X$ females and $X Y$ males (Fig. 2A, Fig. S2). Notably, the transcriptome data showed that many of the female and male PGC-specific expressed genes (FSGs, $n=245 / 651$ ) and (MSGs, $n=157 / 428$ ), which were identified by a previous study (Sakashita et al., 2015), were significantly up- and down-regulated, respectively, in XY female PGCs (Fig. S3, Table S4). Therefore, it is considered that $X Y$ female PGCs, contrary to its genetic sex, have been already switched at this stage from the male property to the female property.

The expression of marker genes for PGCs (Fig. 2B) and gonadal somatic cells (Fig. 2C) showed no global or significant change. However, the expression of POU domain, class 5, transcription factor 1 (Pou5f1), a transcription factor necessary for germ cell differentiation and survival, was significantly up- and

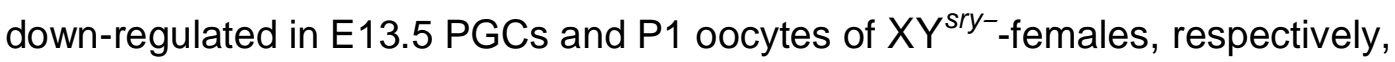
and that deleted in azoospermia-like (Dazl), a RNA binding protein and critical 
for pluripotency maintenance, was significantly decreased in $X Y^{\text {sry--}}{ }^{\text {-PGCs (Fig. }}$ 2B). Furthermore, the expression of forkhead box L2 (Fox/2), a gene encoding a forkhead transcription factor, and that of EGF-like-domain, multiple 6 (Egfl2), encoding a protein with a suggested an adhesive function, from medullary region of the ovary was significantly decreased in $\mathrm{P} 1 \mathrm{XY}^{\text {sry-}}{ }^{-}$-female gonadal somatic cells (GSCs, Fig 2C). These findings demonstrate that the transcriptome datasets obtained were of high quality and valid for subsequent analysis.

Next we focused on differentially expressed genes in E13.5 $X Y^{\text {sry-}}{ }^{-}$-female PGCs, which was screened based on 2 parameters, namely a fold change (FC) of $>2$ and the statistical significance determined using a moderated t-test with a Benjamini-Hochberg FDR of $<0.05$ (Fig 2D). In E13.5 XYsry-female PGCs, 2,537 (21\% of all transcripts) and 2,031 (17\% of all transcripts) genes were upand down-regulated, respectively (Fig 2D and Table S4). While, the number of genes screened as up- and down-regulated was decreased to 411 (3.2\%) and 368 (2.9\%) at the P1 oocytes (Fig 2D and Table S5). Most of the genes, whose expression was significantly changed, were classified to protein-coding cluster (84\%-98\% of all transcripts). Interestingly, proportion of non-coding RNAs (11.3\%) was apparently high in the down-regulated genes of E13.5 $X Y^{\text {sry-}}$-female PGCs (Fig S3A). The 20 genes, which showed the greatest statistical significance in the PGCs and oocytes in $X \mathrm{Y}^{\text {sry-}}{ }^{-}$-females, are also shown in Fig S3B and C. These are reference gene types but the function in germ cells is not well known. Top 3 up-regulated genes of P1 XYsry--female oocytes were expressed from $Y$ chromosome (Fig S3). A few number of differentially expressed genes (16 up-regulated and 69 down-regulated) were

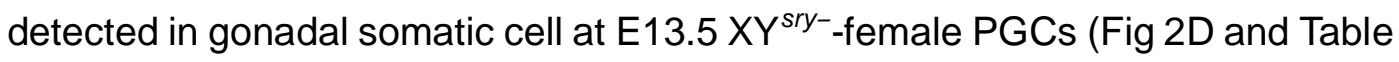
S6). However, the number of the genes greatly increased at P1 stage, especially in up-regulated genes (797 up-regulated and 209 down-regulated, Fig 2D and Table S7). This suggests functional defects of the survived P1 oocytes. These results demonstrated that sex-reversed germ cells are concealed serious transcriptional defects. 
Significant changes of epigenetic modification related genes would affect a large scale change of gene expressions. Then, we examined expressions of DNA methylation and histone medication related genes in E13.5 $X^{\text {sry-}}{ }^{\text {-female }}$ PGCs. No significant change was found in DNA methylation and demethylation related genes, except for Dnmt3b and Tet3, whose expression was very low even in WT female PGCs. However, interestingly, expression of genes, which are related to both active and negative markers of histone modifications, was significantly changed in many cases (Fig. S4). Importantly, expression of Suv39h1, H3K9 methylase, and Kdm4a, H3K9 demethylase, showed an opposite direction change in $X Y^{\text {sry-}}{ }_{-}^{-}$female, which would accelerate H3K9 demethylation of the germ cells. In addition, Kdm6a, H3K27 demethylase was up-regulated and Ring1a, H2AK119 ubiquitin ligase, was down regulated.

\section{Cause of PGC and oocyte depletion}

The number of PGCs at E13.5, when they had reached their maximum (Yokobayashi et al., 2013), was already significantly fewer in $X Y^{\text {sry-}}$-females (Fig. 1C). This observation suggested that the PGCs exhibited inferior proliferation compared with that of $\mathrm{XX}$ females, and also that cell death program was accelerated. Furthermore, the number decreased to $1 / 5$ at the P1 stage in $X Y^{\text {sry-}}$-females compared with $X X$ females. Subsequent analysis of gene ontology (GO) and functional pathways using differentially expressed gene sets provided valuable information toward understanding the mechanisms underlying germ cell depletion in $\mathrm{XY}^{\text {sry-}}{ }^{-}$-females (Table S8 and Figure S5). Upand down-regulated genes were respectively enriched for the following biological processes (Fig. S5A-B): up-regulated genes; "Tissue morphogenesis" and "Enzyme linked receptor protein", down-regulated genes: "DNA metabolism", "Cell cycle process" and "Chromosome organization". GO term interaction analysis formed three large networks regarding "Regulation of gene expression", "Tissue morphogenesis" and "Cell cycle and Cell division" (Fig. $\mathrm{S} 5 \mathrm{C})$.

In particular, the many "Wnt/ $\beta$-catenin signal"-related genes were

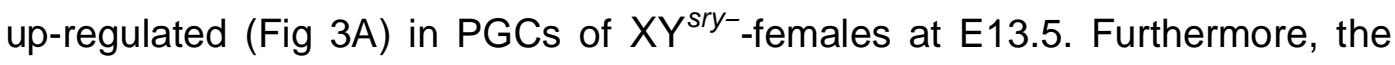


expression of $\beta$-catenin (Ctnnb1) was not changed (Table S2); however, immunofluorescence staining revealed that the proteins were specifically

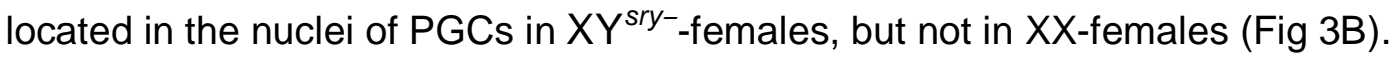
This observation suggests an adverse effect of $\beta$-catenin on cell proliferation. Conversely, the result that many genes related to meiosis were down-regulated suggested that the transition of PGCs to meiosis at E13.5 was delayed in $X Y^{\text {sry-}}$-females (Fig 3C). In addition, Immunofluorescence staining showed that many PGCs in $X Y^{\text {sry-}}$-females expressed the mitosis marker, Ki67 (Fig 3D). Furthermore, apoptosis-related genes, especially the Tnf family, were enhanced (Fig S6A) with tumor necrosis factor receptor superfamily member $12 \mathrm{~A}$ (Tnfrs12a), exhibiting a high degree of elevated expression. Additionally, mRNA expression of the cell death makers Traf, Ripk2 and Caspase tended to increase in $X Y^{\text {sry-}}{ }^{\text {-female }} \mathrm{PGCs}$ as well. However, although the apoptotic cell populations were slightly increased in E13.5 $\mathrm{XY}^{\text {sry--}}{ }^{-P G C s,}$, these were not statistically significant compared with that of WT controls (Figure S6B-C). Unnaturally, many endogenous retrovirus (ERVs) and LINE1 retrotransposons were unlikely highly expressed in E13.5 $\mathrm{XY}^{\text {sry-}}{ }^{-}$-female PGCs (Fig. 3E). ERV1 and satellite DNA were up-regulated in P1 oocytes. Furthermore, a-thalassemia mental retardation X-linked (AtrX), and suppressor of variegation 3-9 homolog 1 (Suv39h1), which are involved in retrotransposable element silencing and chromatin remodelling of heterochromatin structure, were markedly repressed in E13.5 $\mathrm{XY}^{\text {sry-}}$-PGCs (Fig. 3F). These results suggested that oocyte cyst breakdown and primordial follicle formation were considerably disrupted in the neonatal $X Y^{\text {sry- }}{ }^{-}$ovary. Then, we asked whether this aberrant expression of retrotransposable elements led to meiotic defects in the foetal germ cells of $X Y^{\text {sry-}}$-females. Immunofluorescence staining of synaptonemal complex protein 3 (SCP3) showed that the synaptonemal complex between homologous chromosomes was clearly observed in both $X X$ and $X Y^{\text {sry-}}{ }^{-}$-germ cells at E17.5 (Fig. 3G). However, fluorescent signals of phosphorylation of histone H2AX $(\mathrm{YH} 2 \mathrm{AX})$ foci, which indicates unpaired regions, were detected only in the $X Y^{\text {sry- }}{ }^{\text {-germ cells }}(25 / 25 ; 100 \%)$. Furthermore, SCP3 and synaptonemal 
complex protein 1 (SCP1) were well co-localized across a wide range of chromosomes in XX germ cells; in contrast, the co-localization was observed in only a few cases $(6 / 24 ; 24 \%)$ in $X Y^{\text {sry-}}{ }^{-}$-germ cells. Thus, the meiotic process in $X Y^{\text {sry-}}$-germ cells appeared to be disturbed, accompanied with chromosomal asynapsis.

Notably, histological analysis (Fig. 4A) and immunofluorescence staining for DDX4 (Fig. 4B) demonstrated that only a few oocytes were observed in the medullary region of $\mathrm{P} 1 \mathrm{XY}^{\text {sry-}}{ }^{{ }^{-} \text {-ovary. }} \mathrm{MT}$ staining showed that the connective tissues and collagen fibers were proliferated in the medullary region (Fig. 4A). Notably, $X Y^{\text {sry-}}{ }^{-}$-oocytes in the cyst were apparently fewer than those of WT (Fig. 4A) and the proportion of the oocytes in the cyst was significantly high (Fig. 4C). Furthermore, a significantly high proportion of apoptotic oocytes was detected in

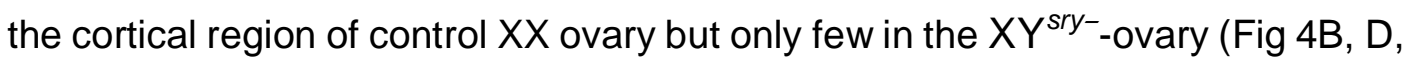
Fig. S7). These observations were supported by transcriptome data. Many

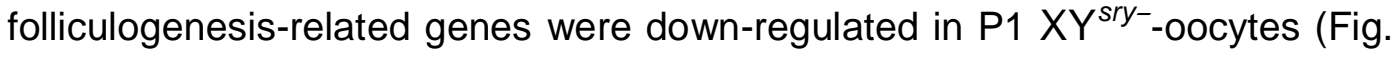
4E). GO analysis showed that differentially expressed genes in the P1 oocytes were enriched in particular annotations, such as "Cell adhesion" and "Cell cycle" (Table S8). Histopathological analysis demonstrated that the primordial oocyte reserve population had almost disappeared from the medulla of the P8

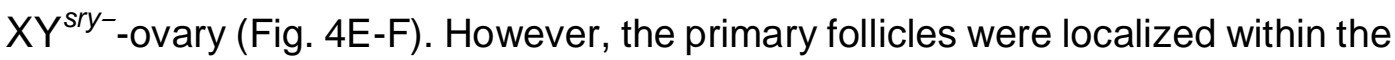
cortical region differ from the XX female ovary.

\section{Y-chromosome-linked gene expressions}

Considering the fertility of $\mathrm{XO}$ mice, it may be considered likely that the dysfunction of Sry deficient $X Y^{\text {sry-}}{ }^{-}$-oocytes is caused by the existence of the $Y$ chromosome itself; i.e., that the expression of genes located on the $Y$ chromosome might present a key factor. Single-copy genes located on $Y$ chromosome MSYp region were expressed at similar levels to those of $X Y$ male germ cells, except some cases (Fig 5B). For example, the expression of Zfy1, Elf2s3y and Uty was significantly low in E13.5 XY ${ }^{\text {sry-}}$-PGCs. The multiple copy genes Rbmy also showed significantly decreased expression in $X^{\text {sry- }}{ }^{\text {-germ }}$ 
cells, especially at P1. These results suggest that the expression of particular genes from the $\mathrm{Y}$ chromosome in $X \mathrm{Y}^{\text {sry-}}{ }^{-}$-females represented a trigger for the depletion and dysfunction of $X Y^{\text {sry-}}{ }^{-}$female germ cells.

\section{Mature oocyte dysfunction}

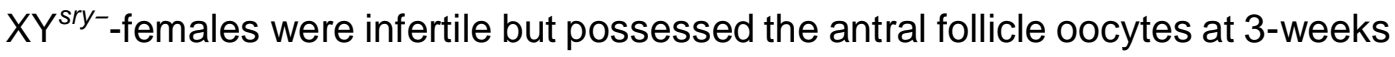
old (Fig. 1E). Therefore, we first examined whether the mutants ovulate mature oocytes in response to exogenous gonadotropins at 4 weeks old. The number of ovulated $X Y^{\text {sry-}}{ }^{-}$oocytes was less than half of the WT $\left(X X: 51.5 \pm 2.5\right.$ vs $X Y^{\text {sry-: }}$ $20.5 \pm 9.1$, Student's $t$-test $P<0.01)$. The Mll oocytes exhibited aberrant spindle formation: diffused poles, skewed spindles, disrupted spindles and misaligned and decondensed chromosomes (Fig. S8B-C). After in vitro fertilization $(n=3)$, $35.6 \%(16 / 32)$ of the MII oocytes showed the capability to fertilize and form male and female pronuclei (Fig. S8D). Of these 10 were arrested at 2-cell stage (Fig. S8D), and one blastocyst obtained but Immunostaining with anti-Oct4 showed failure of differentiation into inner cell mass and trophectoderm cells (Fig. S8E).

To unravel the molecular mechanisms underlying the mature oocyte dysfunction in $X \mathrm{Y}^{\text {sry-}}{ }^{-}$-females, we conducted a single-oocyte transcriptome analysis using $27 X Y^{\text {sry- }}$ and $30 X X$ oocytes. Principal component analysis (PCA) revealed an almost identical gene expression profile in $\mathrm{XX}$ single oocyte libraries

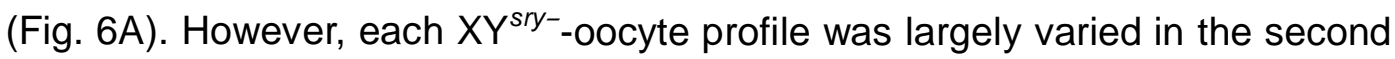
principal component (PC2) (Fig. 6A). The maternal effect genes, which are important for early development, were expressed at levels similar to those of $X X$ oocytes (Fig. 6B). Clustering analysis revealed that $15 \mathrm{XY}^{\text {sry-}}$-oocytes were classified into a cluster that was clearly distinguished from the WT cluster consisting of the 29 oocytes (Fig. 6C). The other mutant oocytes formed a sub-cluster differing from the WT cluster. GO analysis using differentially expressed gene sets in $X Y^{\text {sry-}}$-oocyte, provided the terms related to the oocyte dysfunctions (Fig. 6C); for example, for up-regulated genes: "meiotic cell cycle" and "apoptotic process", down-regulated genes: "protein ubiquitination", "sister chromatid cohesion" and "meiotic nuclear division". Furthermore, interestingly, the retrotransposable elements such as LINE1, ERV and satellite DNA were 
highly expressed in $18 \mathrm{XY}^{\text {sry-}}{ }^{-}$-oocytes, whereas only a single WT oocyte was classified in this cluster (Fig. 6D, Fisher's exact test, $P<0.01$ ). Thus, single oocyte RNA-Seq data demonstrated that most of $X Y^{\text {sry-}}{ }^{-}$MII oocytes exhibited extensive transcriptional anomalies, which might responsible for the observed impairments in the progression of second meiotic division and early development.

\section{Discussion}

Germ cells from any type of sex-reversed mice, i.e., XX males and XY females, exhibit morphologically normal sexual features; however their germ cell development results in failure and inevitably they are infertile (Kato et al., 2013; Lovell-Badge and Robertson, 1990; Ma et al., 2000; Mahadevaiah et al., 1993; Taketo, 2015; Vanderhyden et al., 1997; Vernet et al., 2012; Vernet et al., 2011; Vernet et al., 2014b). Here, we present the first demonstration of the molecular mechanisms underlying germ cell depletion and dysfunction in $X \mathrm{Y}^{\text {sry-}}{ }^{-}$-female mice. In female mice, PGC precursors become committed to differentiate down a female pathway into oocytes between E12.5 and E13.5 (Suzuki et al., 2015), which is typically accompanied by a transition from mitosis to meiosis. From the present integrated analysis of germ cells from $X Y$ sex-reversed females, the complicated mechanisms underlying the observed oocyte depletion in the ovary may be explained by two major distinguishable processes; a decrease of PGCs following the transition from mitosis to meiosis, and a rapid loss of the primordial oocyte pool after birth.

For the first process, it is considered that the $\mathrm{Wnt} / \beta$-catenin signalling pathway would likely be involved in the decline of PGC proliferation in the $X Y^{\text {sry-}}{ }^{-}$females as a large number of genes involving this pathway exhibited significant enhancement. However, although the expression of $\beta$-catenin itself was not increased, it has been reported that the stable localization of $\beta$-catenin at a nucleus of mitotic stage PGCs blocks the transition from G1 to S stage and leads to inhibitory effects on germ cell proliferation in mice (Kimura et al., 2006). Consistent with this observation, fluorescence microscopy clearly showed the predominant nuclear localization of $\beta$-catenin in the $X Y^{\text {sry--female } P G C s .}$ 
Furthermore, the nuclear localization of $\beta$-catenin is inhibited by phosphorylation of exon 3 of this protein, a function catalysed by glycogen synthase kinase $3 \beta$ (GSK-3b) (Wu and Pan, 2010). Therefore, the finding that Gsk3b expression was decreased in $X \mathrm{Y}^{\text {sry-}}{ }^{-}$-female $\mathrm{PGCs}$ suggested that $\beta$-catenin localization into the nuclei of these cells was facilitated likely extensively impacting gene regulation. In tumor cells, overexpression of $\beta$-catenin induce a delay in the cell cycle and apoptosis, independent from the actions of TCF and LEF1 actions (Kim et al., 2000; Olmeda et al., 2003). Therefore, we considered that the Wnt/ $\beta$-catenin signal pathway represented a critical factor towards decreasing the proliferation of PGCs in $X Y^{\text {sry-}}{ }^{-}$females.

In mice, it is known that after PGCs reach to the maximum number $(12,000)$ and enter the prophase of meiosis I at E13.5-14.5, they then enter a continuously decreasing stage resulting in less than one third of the maximum number of cells remaining around the time of birth (Lei and Spradling, 2016; Pepling and Spradling, 2001; Yokobayashi et al., 2013). Evidence has shown that apoptosis represents a major cause of this germ cell reduction. Our previous transcriptome study in mice demonstrated that an intrinsic pathway, such as the mitochondrial pathway, is required to induce the apoptosis of female PGCs; however, the extrinsic pathway was not activated (Sakashita et al., 2015). We also found that the mitochondrial pathway of apoptosis was activated in the PGCs of $X Y^{\text {sry-}}$-females as well. Notably, the present results further demonstrated that in addition to the mitochondrial pathway, an extrinsic apoptosis pathway was also activated in $X \mathrm{Y}^{\text {sry-}}{ }^{-}$-female $\mathrm{PGCs}$, in which the expression of Tnf receptors was significantly enhanced. This additional pathway may therefore promote apoptosis and result in the loss of greater number of oocytes. Furthermore, the enrichment of differentially expressed genes among the 'Response DNA damage stimulus' and 'DNA repair' categories might also have been involved in the germ cell loss. In particular, the down-regulation of Rad51 and Brca, which encode DNA repair enzymes (Scully et al., 1997), in $X Y^{\text {sry-}}$-female PGCs would be expected to induce apoptosis as well. For example, it has been reported that cells mutated for the BRCA proteins lack DNA repairing ability (Yoshida and Miki, 2004), and that Rad51 is involved in the 
resistance to apoptosis following DNA damage, and promotes homologous recombination by chromatid exchange mechanism (Arnaudeau et al., 1999; Xia et al., 1997).

In comparison, in XO female mice, it has been shown that the oocyte number reduced to nearly half compared with that of $X X$ females at neonatal and developmental periods (Burgoyne and Baker, 1981, 1985). Recently, Cloutier et al. (2015) showed that the oocyte elimination in XO mice is linked to the presence of H2AFX serine-139 phosphorylation on the chromatin of asynapsed chromosomes (Cloutier et al., 2015). Therefore, the existence of asynapsed $X Y$ chromosomes may be related to oocyte elimination in sex-reversed females as well. Consistent with this suggestion, we detected $\mathrm{yH} 2 \mathrm{AX}$ foci in all pachytene oocytes from E17.5 $\mathrm{XY}^{\text {sry-}}{ }^{-}$-females. In male spermatogenesis, germ cells that fail to synapse their chromosomes or fail to undergo meiotic sex chromosome inactivation are eliminated by apoptosis during the mid-pachytene stage (Burgoyne et al., 2009; Vernet et al., 2011), and these phenomena were regulated by Zfy2, which is Y-linked transcription regulator (Vernet et al., 2011). This suggests that Zfy2 may be involved in asynapsed pachytene oocyte

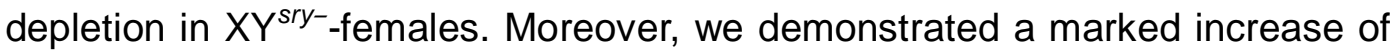
LINE-1 and ERV retrotransposons (>10 fold) in $X Y^{\text {sry-}}{ }^{-}$females, which might have accelerated the germ cell depletion. For example, it has been shown that the forced enhancement of $L 1$ expression is involved in foetal oocyte attrition through increasing the nuclear level of LINE1, which induces a failure of meiotic progression (Malki et al., 2014).

Oocyte loss in the $X \mathrm{Y}^{\text {sry-}}{ }^{-}$-females observed in postnatal stage was drastic and distinctive; thus, the oocyte cyst breakdown may be closely related. It is known that Foxl2 positive medullary granulosa cells are important for precise oocyte cyst breakdown and primordial follicle formation (Tingen et al., 2009; Wang et al., 2017). Knockout of Fox/2 in granulosa cells impairs cyst breakdown by inhibiting granulosa differentiation and proper laying down of the basal lamina around the forming follicle (Uda et al., 2004). In this study, the expression of Foxl2 was significantly decreased in $\mathrm{XY}^{\text {sry-}}{ }^{-}$-female GSCs, compared with WT (Fig. 2C). This might be related to excess proliferated connective tissues and 


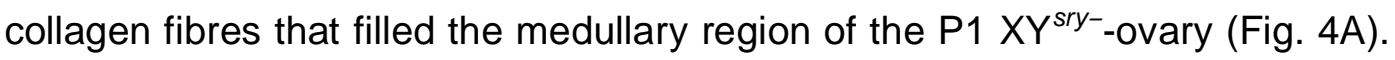
Thus, regression of granulosa cells and the decrease of Fox/2 expression might be involved in impairment of oocyte cyst breakdown and primordial follicle formation in the neonatal $X Y^{\text {sry-}}{ }^{-}$-ovary. Furthermore, it was also reported that Fox/2 deficiency is also associated with premature ovarian failure (Crisponi et al., 2001; Uda et al., 2004). From these, it is supposed that the follicles distributed into the cortical region were aberrantly activated by decreased expression of Fox/2, resulting in the precocious exhaustion of $X Y^{\text {sry- }}{ }^{-0 o c y t e s}$ prior to the pubertal stage.

The fertility of $\mathrm{XO}$ female mice strongly indicates that genes expressed from the $\mathrm{Y}$ chromosome are involved in oocyte depletion in $X \mathrm{Y}^{\text {sry-}}$-females. However, little is known regarding the function of these genes other than Sry. To date, mice harbouring many types of $Y$ chromosome mutation have been reported (Royo et al., 2010; Vernet et al., 2011; Vernet et al., 2014b). Of these, the infertility of $\mathrm{XO}$ mice expressing Zfy2 indicates that Zfy2 can serve as a restricted factor if it is expressed in oocytes (unlike the situation observed in our XY sex-reversed females) (Vernet et al., 2014b). Sex reversed XX male mice harbouring Sry transgene resulted in male infertility consequent to a failure of spermatogenesis (Koopman et al., 1991). However, $\mathrm{X}^{\mathrm{E}} \mathrm{OSry}$ mice carrying the X-linked Eif2s3y gene occasionally led to formation of haploid spermatids, which could be used for round spermatid injection (Vernet et al., 2011; Yamauchi et al., 2014). In addition, Lavery et al. (2011) reported that Sox9 deletion resulted in sex-reversed $X Y$ females that exhibited subfertility in some cases (Lavery et al., 2011), although a comparison between these mice and $X Y^{\text {sry-}}{ }^{-}$-females has not been concluded. These findings suggest that $Y$-linked genes might cause detrimental effects on oogenesis. Accordingly, our transcriptome analysis revealed that the Ube1y1, Eif2s3y, Uty, and Ddx3y genes expressed in XY male PGCs were also expressed at similar levels in $X Y^{\text {sry-}}$-female PGCs (Fig. 5B). From these findings, it might also indicate that if particular $\mathrm{Y}$-linked genes were deleted in addition to Sry, fertile germ cells might be obtained from sex reversed $X Y$ females. 
Furthermore, our single cell transcriptome analysis provided evidence to understand the molecular mechanisms underlying the dysfunction of

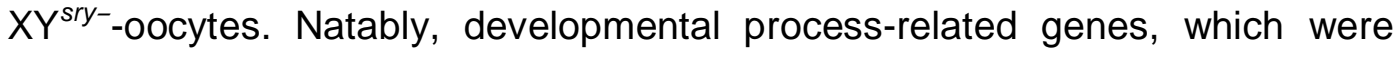
likely to remain transcriptionally silent in germline cells through bivalent chromatin marks with H3K4me3 and H3K27me3 (Hammoud et al., 2009; Reik, 2007; Sachs et al., 2013), were activated in $X Y^{\text {sry-}}-$ MIl oocytes (Fig. 6C). Moreover, 6 genes (Zfy1, Ube1y1, Kdm5d, Uty, Ddx3y, Zfy2) located at MSYp region also expressed in $X \mathrm{Y}^{\text {sry-}}{ }^{-}$-mature $\mathrm{MIl}$ oocytes. As a result of $\mathrm{GO}$ analysis for respective using the DAVID web tool (Wang et al., 2013), it was revealed that $D d x 3 y$ and Eif2s3y had the functions associated with "chromosome segregation" and "translation", respectively. Furthermore, it had been reported that the expression of Zfy2 in $X Y$ oocytes elicited frequent MII defects and preimplantation developmental arrest.

In conclusion, the present study conducted inclusive transcriptome and bioinformatics analysis and demonstrated that the serious germ cell loss in $X Y$ sex-reversed female mice is induced by a decline of cell proliferation, an acceleration of cell death programs and follicle formation. Furthermore, we showed that the failure of DNA remodeling and repair in $X Y^{\text {sry--female oocytes }}$ resulted in germ cell loss and dysfunction. These results may provide further insight into understanding disorders of sex development in human, such as Swyer syndrome, wherein the patients are anatomically females but have an $X Y$ karyotype and exhibited infertility consequent to mutations in Sry and Map3k1 (Pearlman et al., 2010).

\section{Materials and Methods}

\section{Animals and ethic statements}

This study was carried out in strict accordance with the Tokyo University of Agriculture Guide for Care and Use of Laboratory Animals. The protocol was approved by the Committee on the Ethics of Animal Experiments of the Tokyo University of Agriculture (Permit Number: 260064SE). At the time of sample collection, all animals (CLEA JAPAN, JP) were sacrificed by cervical dislocation, and all efforts were made to minimize suffering. 


\section{Generation of Sry mutant mice (XYsry--females) by CRISPR/Cas9 system}

To generate the Sry targeting vector, we used a pX330-U6-Chimeric BB-CBh-hSpCas9 (pX330) plasmid, kindly provided by Dr. Ikawa (Research Institute for Microbial Diseases, Osaka University). sgRNA sequence was designated on SRY HMG-box domain (5'- TGGTGTGGTCCCGTGGTGAG -3'), and inserted annealed double strand DNA with 4 overhangs into Bbsl site in pX330 plasmid. B6 female mice were superovulated and mated B6 male mice, and zygotes were collected from ampulla of the oviducts. The microinjection of the Sry targeting pX330 plasmid (2 $\mathrm{ng} / \mathrm{\mu l}$ ) into male pronuclear of PN3-5 stage zygotes was carried out under standard procedures. The injected zygotes were cultured in potassium simplex optimized medium (KSOM) at $37^{\circ} \mathrm{C}$ and embryos developed into the two-cell stage were transferred into oviducts of pseudopregnant ICR female mice.

\section{PCR-based genotyping and sexing}

Genomic DNA was extracted from heads or tail tips using $\mathrm{NaOH}$ extraction method (Wang et al., 1993). For genotyping of Sry, PCR was carried out using specific primer sets: Sry_F (5'- GTGACAATTGTCTAGAGAGCATGGAGG -3') and Sry_R (5' - TCCAGTCTTGCCTGTATGTGAT -3'). The PCR products were purified by QIAquick PCR purification kit (QIAGEN, Hilden, FRG) and used as templates for sequencing. Sanger sequencing was done by ABI PRISM 3100 Genetic Analyzer (Applied Biosystems, CA, USA). For PCR-based sexing, $X$ chromosome specific gene (Xist) and $Y$ chromosome specific gene (Zfy) were amplified using following primer sets (Kay et al., 1994; Zuccotti and Monk, 1995):

Xist_F; 5'-AGGATAATCCTTCATTATCGCGC -3',

Xist_R; 5'-AAACGAGCAAACATGGCTGGAG -3'

Zfy_F; 5'-GACTAGACATGTCTTAACATCTGTCC -3'

Zfy_R; 5'- CCTATTGCATGGACAGCAGCTTATG -3'

\section{Fertility and development test}


$X Y^{\text {sry-}}{ }^{-}$females, 8-12 weeks old, were mated with fertile B6 males. The presence of the vaginal plug was checked every morning for four weeks. MII oocytes were collected by PMSG-hCG treatment from 4-week-old mice, and the ovulated oocytes were applied to IVF. The zygotes obtained were subjected to in vitro culture for 4 days in KSOM.

\section{Germ cells and gonadal somatic cells collection}

To collect E13.5 PGCs, P1 oocytes and gonadal somatic cells (GSCs), gonads from $13.5 \mathrm{dpc}$ and $1 \mathrm{dpp}$ after embryo transferred were digested $1 \mathrm{mg} / \mathrm{ml}$ collagenase solution (Wako, Oosaka, JP) at $37^{\circ} \mathrm{C}$ for $40 \mathrm{~min}$, followed by treatment with $0.25 \%$ trypsin-EDTA solution (0.53 mM; Sigma, MO, USA) at $37^{\circ} \mathrm{C}$ for $15 \mathrm{~min}$. After adding foetal bovine serum (FBS), a single-cell suspension was obtained by gentle pipetting. Cells were then incubated in a 1:50 dilution of PE-conjugated anti-SSEA1 (for labeled E13.5 PGCs, 560142, BD Pharmingen, NJ, USA) or PE-conjugated anti-CD117 (for labeled P1 oocytes, 105807, BioLegend, CA, USA) antibody. PE positive cells (PGCs and oocytes) and negative cells (Gonadal somatic cells) were isolated and collected using a FACSAria II cell sorter (BD Bioscience, NJ, USA, Fig. S9). Ovulated MII oocytes were extracted from the oviducts of $X X$ and $X Y^{\text {sry-}}{ }^{-}$female mice according to methods described in our previous report (Obata and Kono, 2002).

Considering the variability in the genotype of the $X Y^{\text {sry--females, when }}$ collecting PGCs, oocytes, gonadal somatic cells and MII oocytes, we supplied pooled gonadal cell suspension and MII oocytes from 4 and $5 X^{X^{\text {sry-}}{ }^{-} \text {-female }}$ individuals at each time point. The Sry mutation types are listed below. Furthermore, we confirmed that all mutant lines contained a frameshift mutation within HMG-box coding region, by converting DNA nucleotide sequences to amino acid sequences, and thus were expected to cause Sry protein disruption.

\section{【E13.5: 4 samples】}

\#1: 5 bp deletion

\section{ATGGAGGGCCATGTCAAGCGCCCCATGAATGCATTTATGGTGTGGTCCCG TGG----AGGCACAAG}

\#2 and \#3: 7 bp deletion: 
ATGGAGGGCCATGTCAAGCGCCCCATGAATGCATTTATGGTGTGGTCCCG TGGT-------CACAAG

\#4: 1 bp insertion

ATGGAGGGCCATGTCAAGCGCCCCATGAATGCATTTATGGTGTGGTCCCG TGGTIGAGAGGCACAAG

[P1: 4 samples】

\#1 and \#2: 1 bp insertion

ATGGAGGGCCATGTCAAGCGCCCCATGAATGCATTTATGGTGTGGTCCCG TGGTIGAGAGGCACAAG

\#3: 14 bp deletion

ATGGAGGGCCATGTCAAGCGCCCCATGAATGCATTTATGGTGTGGTCCCG TG---------G

\#4: 7 bp deletion

ATGGAGGGCCATGTCAAGCGCCCCATGAATGCATTTATGGTGTGGTCCCG TGGT------CACAAG

【MII oocytes: 5 samples】

\#1, \#2 and \#3: 1 bp insertion

ATGGAGGGCCATGTCAAGCGCCCCATGAATGCATTTATGGTGTGGTCCCG TGGTIGAGAGGCACAAG

\#4: 8 bp deletion

ATGGAGGGCCATGTCAAGCGCCCCATGAATGCATTTATGGTGTGGTCCCG ------GGCACAAG

\#5: 2bp insertion

ATGGAGGGCCATGTCAAGCGCCCCATGAATGCATTTATGGTGTGGTCCCG TGGTㄷGAGAGGCACAAG

\section{RNA isolation, RNA-Seq library preparation and sequencing}

Total RNA of PGCs, oocytes and GSCs was isolated using an RNeasy Micro Kit (QIAGEN, Hilden, FRG) with DNase treatment. cDNA synthesis and pre-amplification were performed with total RNA (10 ng) using a SMARTer Ultra Low Input RNA Kit and an Advantage 2 PCR Kit (Clontech, CA, USA), respectively, according to the manufacturers' instructions. Pre-amplified cDNA 
was fragmented into 200-bp fragments using an S2 sonicator (Covaris, MA, USA) and then used to construct sequencing libraries using a NEBNext Ultra DNA Library Prep Kit, according to the manufacturers' protocol (New England BioLabs, MA, USA). Two biological replicates were used for each sample.

To perform single cell RNA-Seq analysis of 4-weeks-old $X X$ and $X Y^{\text {sry-}}{ }^{-M I I}$ oocytes, single and bulk ( $n=20-22$ ) MIl oocytes from oviducts of the respective genotypes were picked by glass pipette and were immediately lysed in Clontech Lysis Buffer (Mountain View, CA, USA). The whole lysate was subjected to cDNA synthesis and RNA-Seq library using a SMARTer Ultra Low Input RNA Kit (Clontech) and Nextera XT library prep kit, according to manufacturer's instruction (Illumina, San Diego, CA, USA). Indexed libraries were pooled (10 $\mathrm{nM}$ each), and sequenced using an Illumina Hiseq2500 sequencer (single-end, 100 bp condition).

\section{RNA-Seq alignments and statistical analysis}

RNA-Seq reads for each sample were aligned to the mouse genome (mm10, Genome Reference Consortium Mouse Build 38) with the CLC Genomics Workbench (QIAGEN, Hilden, FRG). Aligned reads were subsequently assembled into transcripts guided by reference annotation ( $\mathrm{mm} 10$, UCSC gene annotation). Transcript expression was quantified in terms of reads per million mapped reads and normalized using RPKM method with Strand NGS (Agilent, CA, USA). To identify significant differentially expressed genes between $X X$ female vs $X Y^{\text {sry-}}{ }^{-}$female E13.5 PGCs and P1 oocytes, we used 2 selection criteria. The first criterion was a fold-change in expression of at least two-fold. The second criterion was removal of false positive genes using a moderated $t$-test with Benjamini-Hochberg false-discovery rate (FDR) of <0.05). Differentially expressed gene lists were used for GO analysis with the DAVID web tool (http://david.abcc.ncifcrf.gov/) (Huang da et al., 2009); a background of all mouse genes was applied. Biological-process term groups with a significance of $P<0.01$ (modified Fisher's exact test) were considered significant.

Single oocyte transcriptome datasets were applied to PCA and k-means cluster analysis to identify common up and downregulated genes in 
$X Y^{\text {sry-}-o o c y t e s ~ g r o u p s . ~ R e s p e c t i v e ~ g e n e ~ e x p r e s s i o n ~ p r o f i l e s ~ w e r e ~ s u b j e c t e d ~ t o ~}$ bi-dimensional PCA using $\mathrm{R}$ prcomp function and ggplot2 package (https://www.r-project.org/)

Each sample score from the covariance matrix was plotted in the two eigenvectors PC1 and PC2. To determine the optimum number of k-means clusters, we used the hierarchical cluster datasets of each gene, and further grouped the determined $4 \mathrm{k}$-means subclusters. The first subcluster comprised genes that showed common upregulated genes $(n=902)$ in $X Y^{\text {sry-}}{ }^{-M I I}$ oocytes. The second subcluster comprised genes showing common down-regulated genes $(n=874)$ in $X Y^{\text {sry-}}$-MII oocytes. In contrast, other two subclusters (third and fourth subcluster) comprised genes that showed uniformly expression pattern between $X X$ and $X Y^{\text {sry-}}$-MIl oocytes. We performed $\mathrm{GO}$ analysis of common up and down-regulated gens using the DAVID web tool.

\section{Transposable elements analysis}

The current build of rodent repeat sequences was downloaded from Repbase (http://www.girinst.org/repbase/) and filtered for Mus musculus sequences. Raw RNA-Seq reads were aligned to the repetitive sequence database by CLC Genomics Workbench (QIAGEN, Hilden, FRG). Aligned reads were subsequently assembled into transcripts guided by reference annotation. Transcript expression was quantified in terms of reads per million mapped reads and normalized using RPKM method with Strand NGS (Agilent, CA, USA).

\section{Tissue collection, histological analysis and TUNEL assay}

We fixed freshly dissected ovaries in 4\% paraformaldehyde in PBS (-) at room temperature for overnight. The following day, fixed tissue samples were subjected to paraffin sectioning, hematoxylin and eosin (HE) staining and Masson's trichrime (MT) staining. Also, TUNEL positive oocytes were detected in paraffin sections of P1 ovaries using the anti-DDX4 antibody (1/300 dilution, ab13840, Abcam, Cambridge, UK) and In Situ Cell Death Detection Kit, Fluorescein (11684795910, Roche, Basel, Switzerland). 


\section{Germ cell counts}

The number of germ cells was determined for each day of development from $13.5 \mathrm{dpc}, 1 \mathrm{dpp}$ and $8 \mathrm{dpp}$. First, to visualize germ cell, each whole ovary was stained sequentially first with anti-DDX4 antibody (ab13840, Abcam, Cambridge, UK) then the anti-rabbit Alexa 568 secondary antibody. Second, the number of DDX4 positive cells in a single representative section was counted. Total number of germ cells in one fraction was calculated using stacks of confocal sections (LSM710, Zeiss, Oberkohen, FRG).

\section{Immunofluorescence staining}

Whole gonads were fixed in 4\% paraformaldehyde in PBS (-) for $1 \mathrm{hr}$, and then incubated for 2 days at $4{ }^{\circ} \mathrm{C}$ with blocking solution (10\% FBS, 3\% BSA, 0.2\% TritonX-100 in PBS (-)). Whole mount immunofluorescence analysis conducted using the following primary antibodies: anti- $\beta$-catenin (1/200 dilution, 610153, BD Transduction Laboratories, NJ, USA), anti-Ki67 (1/200 dilution, 556003, BD Pharmingen, NJ, USA), anti-cCASP3 (1/200 dilution, 9661, CST, MA, USA), anti-cPARP (1/200 dilution, 9544, CST, MA, USA) and anti-TRA98 (1/200 dilution, 73-003, BioAcademia, Oosaka, Japan). Gonads were incubated for 3 days at $4{ }^{\circ} \mathrm{C}$ with primary antibody, and then washed and incubated with Alexa Fluor 488- or 568-conjugated anti-rabbit, anti-mouse or anti-rat IgG secondary antibody (1/500 dilution, Life technologies, CA, USA) for 2 days at $4 \stackrel{\circ}{ } \mathrm{C}$. The slides were mounted in Prolong Antifade Medium containing DAPI (Molecular Probe, MA, USA).

Ovarian cell suspensions were fixed in 1\% paraformaldehyde in PBS (-) for 1 hr. Cells were washed PBS (-), and incubated overnight at room temperature with blocking solution. Immunofluorescence analysis of respectively proteins in ovarian cells conducted using the following primary antibodies: anti-yH2AX (1/200 dilution, ab11174, Abcam, Cambridge, UK), anti-SCP3 (1/500 dilution, ab97672, Abcam, Cambridge, UK) and anti-SCP1 (1/500 dilution, ab15090, Abcam, Cambridge, UK). Cells were incubated overnight at room temperature with primary antibody, and then washed and incubated with Alexa Fluor 488- or 568-conjugated anti-rabbit or anti-mouse lgG secondary antibody (1/500 dilution, 
Life technologies, CA, USA) at room temperature for $1 \mathrm{hr}$. The slides were mounted in Prolong Antifade Medium containing DAPI (Molecular Probe, MA, USA). Fluorescent signals were detected and quantified under the LSM710 confocal microscopy (Zeiss, Oberkohen, FRG). For all the observation, exposure time was kept the same between $X X$ and $X Y$ female samples.

\section{Data from other source}

Previously published RNA-Seq data from mouse E13.5 female and male PGCs (DRA003597), and P1 spermatogonial stem cells (DRA002477) were downloaded from DNA Data Bank of Japan (DDBJ), and aligned to allow comparison with the present data.

\section{Accession number}

The RNA-Seq data from this study have been deposited in the DDBJ under the accession number DRA005345 and DRA005822.

\section{Acknowledgments}

We thank Hidehiko Ogawa, Yayoi Obata, Hisato Kobayashi for helpful comments, and Asuka Kamio, Takumi Yoshioka for assistance of NGS data collection and data analysis.

\section{Author Contributions}

T. Kono and A. Sakashita conceived and designed the experiments. T. Wakai provided instructions regarding the construction of the CRISPR/Cas9 targeting plasmid. A. Sakashita, C. Nishimura and Y. Sotomaru performed microinjection experiments, and carried out the phenotypic analysis of generated $X Y^{\text {sry-}}$-females. A. Sakashita and Y. Kawabata performed RNA-Seq and data analysis. T. Kono and A. Sakashita wrote the paper. All authors discussed the results and commented on the manuscript. 


\section{Competing Financial Interests}

The authors declare no competing financial interests.

\section{References}

Arnaudeau, C., Helleday, T., and Jenssen, D. (1999). The RAD51 protein supports homologous recombination by an exchange mechanism in mammalian cells. J Mol Biol 289, 1231-1238.

Burgoyne, P.S., and Baker, T.G. (1981). Oocyte depletion in XO mice and their XX sibs from 12 to 200 days post partum. J Reprod Fertil 61, 207-212.

Burgoyne, P.S., and Baker, T.G. (1985). Perinatal oocyte loss in XO mice and its implications for the aetiology of gonadal dysgenesis in XO women. J Reprod Fertil 75, 633-645.

Burgoyne, P.S., Mahadevaiah, S.K., and Turner, J.M. (2009). The consequences of asynapsis for mammalian meiosis. Nat Rev Genet 10, 207-216.

Cloutier, J.M., Mahadevaiah, S.K., Ellnati, E., Nussenzweig, A., Toth, A., and Turner, J.M. (2015). Histone H2AFX Links Meiotic Chromosome Asynapsis to Prophase I Oocyte Loss in Mammals. PLoS Genet 11, e1005462.

Correa, S.M., Washburn, L.L., Kahlon, R.S., Musson, M.C., Bouma, G.J., Eicher, E.M., and Albrecht, K.H. (2012). Sex reversal in C57BL/6J XY mice caused by increased expression of ovarian genes and insufficient activation of the testis determining pathway. PLoS Genet 8, e1002569.

Coward, P., Nagai, K., Chen, D., Thomas, H.D., Nagamine, C.M., and Lau, Y.F. (1994). Polymorphism of a CAG trinucleotide repeat within Sry correlates with B6.YDom sex reversal. Nat Genet 6, 245-250.

Crisponi, L., Deiana, M., Loi, A., Chiappe, F., Uda, M., Amati, P., Bisceglia, L., Zelante, L., Nagaraja, R., Porcu, S., et al. (2001). The putative forkhead transcription factor FOXL2 is mutated in blepharophimosis/ptosis/epicanthus inversus syndrome. Nat Genet 27, 159-166.

Eicher, E.M., Washburn, L.L., Whitney, J.B., 3rd, and Morrow, K.E. (1982). Mus poschiavinus $\mathrm{Y}$ chromosome in the C57BL/6J murine genome causes sex reversal. Science 217, 535-537. 
Gubbay, J., Collignon, J., Koopman, P., Capel, B., Economou, A., Munsterberg, A., Vivian, N., Goodfellow, P., and Lovell-Badge, R. (1990). A gene mapping to the sex-determining region of the mouse $Y$ chromosome is a member of a novel family of embryonically expressed genes. Nature 346, 245-250.

Gubbay, J., Vivian, N., Economou, A., Jackson, D., Goodfellow, P., and Lovell-Badge, R. (1992). Inverted repeat structure of the Sry locus in mice. Proc Natl Acad Sci U S A 89, 7953-7957.

Hammoud, S.S., Nix, D.A., Zhang, H., Purwar, J., Carrell, D.T., and Cairns, B.R. (2009). Distinctive chromatin in human sperm packages genes for embryo development. Nature 460, 473-478.

Huang da, W., Sherman, B.T., and Lempicki, R.A. (2009). Bioinformatics enrichment tools: paths toward the comprehensive functional analysis of large gene lists. Nucleic Acids Res 37, 1-13.

Jiang, J.F., Tian, Q.J., Xue, W., Deng, Y., Zheng, T.P., and Sun, A.J. (2016). Clinical Features of 32 Patients with XO/XY Gonadal Dysgenesis. Zhongguo Yi Xue Ke Xue Yuan Xue Bao 38, 411-414.

Kashimada, K., and Koopman, P. (2010). Sry: the master switch in mammalian sex determination. Development 137, 3921-3930.

Kato, T., Miyata, K., Sonobe, M., Yamashita, S., Tamano, M., Miura, K., Kanai, Y., Miyamoto, S., Sakuma, T., Yamamoto, T., et al. (2013). Production of Sry knockout mouse using TALEN via oocyte injection. Sci Rep 3, 3136.

Kay, G.F., Barton, S.C., Surani, M.A., and Rastan, S. (1994). Imprinting and X chromosome counting mechanisms determine Xist expression in early mouse development. Cell 77, 639-650.

Kim, K., Pang, K.M., Evans, M., and Hay, E.D. (2000). Overexpression of beta-catenin induces apoptosis independent of its transactivation function with LEF-1 or the involvement of major G1 cell cycle regulators. Mol Biol Cell 11, 3509-3523.

Kimura, T., Nakamura, T., Murayama, K., Umehara, H., Yamano, N., Watanabe, S., Taketo, M.M., and Nakano, T. (2006). The stabilization of beta-catenin leads to impaired primordial germ cell development via aberrant cell cycle progression. Dev Biol 300, 545-553. 
Koopman, P., Gubbay, J., Vivian, N., Goodfellow, P., and Lovell-Badge, R. (1991). Male development of chromosomally female mice transgenic for Sry. Nature 351, 117-121.

Kuroki, S., Matoba, S., Akiyoshi, M., Matsumura, Y., Miyachi, H., Mise, N., Abe, K., Ogura, A., Wilhelm, D., Koopman, P., et al. (2013). Epigenetic regulation of mouse sex determination by the histone demethylase Jmjd1a. Science 341, 1106-1109.

Lavery, R., Lardenois, A., Ranc-Jianmotamedi, F., Pauper, E., Gregoire, E.P., Vigier, C., Moreilhon, C., Primig, M., and Chaboissier, M.C. (2011). XY Sox9 embryonic loss-of-function mouse mutants show complete sex reversal and produce partially fertile XY oocytes. Dev Biol 354, 111-122.

Lee, C.H., and Taketo, T. (1994). Normal onset, but prolonged expression, of Sry gene in the B6.YDOM sex-reversed mouse gonad. Dev Biol 165, 442-452.

Lei, L., and Spradling, A.C. (2016). Mouse oocytes differentiate through organelle enrichment from sister cyst germ cells. Science 352, 95-99.

Lovell-Badge, R., and Robertson, E. (1990). XY female mice resulting from a heritable mutation in the primary testis-determining gene, Tdy. Development 109, 635-646.

Ma, K., Mallidis, C., and Bhasin, S. (2000). The role of $Y$ chromosome deletions in male infertility. Eur J Endocrinol 142, 418-430.

Mahadevaiah, S.K., Lovell-Badge, R., and Burgoyne, P.S. (1993). Tdy-negative $X Y, X X Y$ and $X Y Y$ female mice: breeding data and synaptonemal complex analysis. J Reprod Fertil 97, 151-160.

Malki, S., van der Heijden, G.W., O'Donnell, K.A., Martin, S.L., and Bortvin, A. (2014). A role for retrotransposon LINE-1 in fetal oocyte attrition in mice. Dev Cell 29, 521-533.

Matsubara, Y., Kato, T., Kashimada, K., Tanaka, H., Zhi, Z., Ichinose, S., Mizutani, S., Morio, T., Chiba, T., Ito, Y., et al. (2015). TALEN-Mediated Gene Disruption on $\mathrm{Y}$ Chromosome Reveals Critical Role of EIF2S3Y in Mouse Spermatogenesis. Stem Cells Dev 24, 1164-1170.

Miyamoto, Y., Taniguchi, H., Hamel, F., Silversides, D.W., and Viger, R.S. (2008). A GATA4/WT1 cooperation regulates transcription of genes required for 
mammalian sex determination and differentiation. BMC Mol Biol 9, 44.

Obata, Y., and Kono, T. (2002). Maternal primary imprinting is established at a specific time for each gene throughout oocyte growth. J Biol Chem 277, 5285-5289.

Olmeda, D., Castel, S., Vilaro, S., and Cano, A. (2003). Beta-catenin regulation during the cell cycle: implications in G2/M and apoptosis. Mol Biol Cell 14, 2844-2860.

Park, E.H., and Taketo, T. (2003). Onset and progress of meiotic prophase in the oocytes in the B6.YTIR sex-reversed mouse ovary. Biol Reprod 69, 1879-1889. Pearlman, A., Loke, J., Le Caignec, C., White, S., Chin, L., Friedman, A., Warr, N., Willan, J., Brauer, D., Farmer, C., et al. (2010). Mutations in MAP3K1 cause $46, X Y$ disorders of sex development and implicate a common signal transduction pathway in human testis determination. Am J Hum Genet 87, 898-904.

Pepling, M.E., and Spradling, A.C. (2001). Mouse ovarian germ cell cysts undergo programmed breakdown to form primordial follicles. Dev Biol 234, 339-351.

Probst, F.J., Cooper, M.L., Cheung, S.W., and Justice, M.J. (2008). Genotype, phenotype, and karyotype correlation in the $\mathrm{XO}$ mouse model of Turner Syndrome. J Hered 99, 512-517.

Reik, W. (2007). Stability and flexibility of epigenetic gene regulation in mammalian development. Nature 447, 425-432.

Royo, H., Polikiewicz, G., Mahadevaiah, S.K., Prosser, H., Mitchell, M., Bradley, A., de Rooij, D.G., Burgoyne, P.S., and Turner, J.M. (2010). Evidence that meiotic sex chromosome inactivation is essential for male fertility. Curr Biol 20, 2117-2123.

Sachs, M., Onodera, C., Blaschke, K., Ebata, K.T., Song, J.S., and Ramalho-Santos, M. (2013). Bivalent chromatin marks developmental regulatory genes in the mouse embryonic germline in vivo. Cell Rep 3, 1777-1784.

Sakashita, A., Kawabata, Y., Jincho, Y., Tajima, S., Kumamoto, S., Kobayashi, H., Matsui, Y., and Kono, T. (2015). Sex Specification and Heterogeneity of 
Primordial Germ Cells in Mice. PLoS One 10, e0144836.

Scully, R., Chen, J., Plug, A., Xiao, Y., Weaver, D., Feunteun, J., Ashley, T., and Livingston, D.M. (1997). Association of BRCA1 with Rad51 in mitotic and meiotic cells. Cell 88, 265-275.

Suzuki, H., Kanai-Azuma, M., and Kanai, Y. (2015). From Sex Determination to Initial Folliculogenesis in Mammalian Ovaries: Morphogenetic Waves along the Anteroposterior and Dorsoventral Axes. Sex Dev 9, 190-204.

Taketo, T. (2015). The role of sex chromosomes in mammalian germ cell differentiation: can the germ cells carrying $X$ and $Y$ chromosomes differentiate into fertile oocytes? Asian J Androl 17, 360-366.

Tam, Y.H., Wong, Y.S., Pang, K.K., To, K.F., Yiu, A.K., Wong, H.Y., Tsui, S.Y., Mou, J.W., Chan, K.W., and Lee, K.H. (2016). Tumor risk of children with $45, X / 46, X Y$ gonadal dysgenesis in relation to their clinical presentations: Further insights into the gonadal management. J Pediatr Surg 51, 1462-1466.

Tanaka, S.S., and Nishinakamura, R. (2014). Regulation of male sex determination: genital ridge formation and Sry activation in mice. Cell Mol Life Sci 71, 4781-4802.

Tingen, C., Kim, A., and Woodruff, T.K. (2009). The primordial pool of follicles and nest breakdown in mammalian ovaries. Mol Hum Reprod 15, 795-803.

Uda, M., Ottolenghi, C., Crisponi, L., Garcia, J.E., Deiana, M., Kimber, W., Forabosco, A., Cao, A., Schlessinger, D., and Pilia, G. (2004). Foxl2 disruption causes mouse ovarian failure by pervasive blockage of follicle development. Hum Mol Genet 13, 1171-1181.

Vanderhyden, B.C., Macdonald, E.A., Merchant-Larios, H., Fernandez, A., Amleh, A., Nasseri, R., and Taketo, T. (1997). Interactions between the oocyte and cumulus cells in the ovary of the B6.Y(TIR) sex-reversed female mouse. Biol Reprod 57, 641-646.

Vernet, N., Mahadevaiah, S.K., Ellis, P.J., de Rooij, D.G., and Burgoyne, P.S. (2012). Spermatid development in XO male mice with varying Y chromosome short-arm gene content: evidence for a $Y$ gene controlling the initiation of sperm morphogenesis. Reproduction 144, 433-445.

Vernet, N., Mahadevaiah, S.K., Ojarikre, O.A., Longepied, G., Prosser, H.M., 
Bradley, A., Mitchell, M.J., and Burgoyne, P.S. (2011). The Y-encoded gene zfy2 acts to remove cells with unpaired chromosomes at the first meiotic metaphase in male mice. Curr Biol 21, 787-793.

Vernet, N., Mahadevaiah, S.K., Yamauchi, Y., Decarpentrie, F., Mitchell, M.J., Ward, M.A., and Burgoyne, P.S. (2014a). Mouse Y-linked Zfy1 and Zfy2 are expressed during the male-specific interphase between meiosis I and meiosis II and promote the 2nd meiotic division. PLoS Genet 10, e1004444.

Vernet, N., Szot, M., Mahadevaiah, S.K., Ellis, P.J., Decarpentrie, F., Ojarikre, O.A., Rattigan, A., Taketo, T., and Burgoyne, P.S. (2014b). The expression of Y-linked Zfy2 in XY mouse oocytes leads to frequent meiosis 2 defects, a high incidence of subsequent early cleavage stage arrest and infertility. Development $141,855-866$.

Villemure, M., Chen, H.Y., Kurokawa, M., Fissore, R.A., and Taketo, T. (2007). The presence of $\mathrm{X}$ - and $\mathrm{Y}$-chromosomes in oocytes leads to impairment in the progression of the second meiotic division. Dev Biol 301, 1-13.

Wang, C., Zhou, B., and Xia, G. (2017). Mechanisms controlling germline cyst breakdown and primordial follicle formation. Cell Mol Life Sci.

Wang, H., Hu, Y.C., Markoulaki, S., Welstead, G.G., Cheng, A.W., Shivalila, C.S., Pyntikova, T., Dadon, D.B., Voytas, D.F., Bogdanove, A.J., et al. (2013). TALEN-mediated editing of the mouse $Y$ chromosome. Nat Biotechnol 31, 530-532.

Wang, H., Qi, M., and Cutler, A.J. (1993). A simple method of preparing plant samples for PCR. Nucleic Acids Res 21, 4153-4154.

Washburn, L.L., Albrecht, K.H., and Eicher, E.M. (2001). C57BL/6J-T-associated sex reversal in mice is caused by reduced expression of a Mus domesticus Sry allele. Genetics 158, 1675-1681.

Wong, J., Luckers, L., Okawara, Y., Pelletier, R., and Taketo, T. (2000). Follicular development and atresia in the B6.Y(TIR) sex-reversed mouse ovary. Biol Reprod 63, 756-762.

Wu, D., and Pan, W. (2010). GSK3: a multifaceted kinase in Wnt signaling. Trends Biochem Sci 35, 161-168.

Xia, S.J., Shammas, M.A., and Shmookler Reis, R.J. (1997). Elevated 
recombination in immortal human cells is mediated by HsRAD51 recombinase. Mol Cell Biol 17, 7151-7158.

Yamauchi, Y., Riel, J.M., Stoytcheva, Z., and Ward, M.A. (2014). Two Y genes can replace the entire $Y$ chromosome for assisted reproduction in the mouse. Science 343, 69-72.

Yokobayashi, S., Liang, C.Y., Kohler, H., Nestorov, P., Liu, Z., Vidal, M., van Lohuizen, M., Roloff, T.C., and Peters, A.H. (2013). PRC1 coordinates timing of sexual differentiation of female primordial germ cells. Nature 495, 236-240.

Yoshida, K., and Miki, Y. (2004). Role of BRCA1 and BRCA2 as regulators of DNA repair, transcription, and cell cycle in response to DNA damage. Cancer Sci 95, 866-871.

Zhong, Q., and Layman, L.C. (2012). Genetic considerations in the patient with Turner syndrome--45,X with or without mosaicism. Fertil Steril 98, 775-779.

Zuccotti, M., and Monk, M. (1995). Methylation of the mouse Xist gene in sperm and eggs correlates with imprinted Xist expression and paternal X-inactivation. Nat Genet 9, 316-320. 


\section{Figure legends}

Fig. 1. $X Y^{\text {sry-}}{ }^{-}$-female mice exhibit infertility and oocyte depletion.

(A) External genitalia and internal reproductive organs of an XX wild-type (WT)

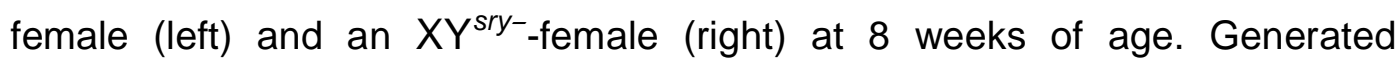

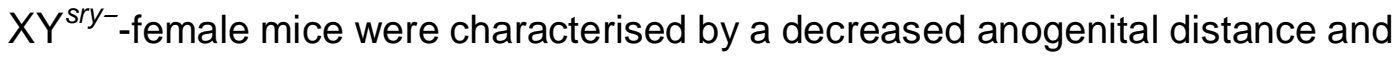
internal reproductive structure, comprised of a converted ovary. (B) Fertility test of XXWT and $X Y^{\text {sry-}}$-females. Left bar graph shows the total number of checked plugs; right bar graph illustrates the total number of pregnancies in each genotype. Number of tested females of each genotype is shown at the bottom of the respective bars. Pink and blue bars indicate WT and $X Y^{\text {sry-}}{ }^{\text {-females, }}$ respectively. (C) Boxplot of the total oocyte number per 1 fraction (x: $212.2 \mu \mathrm{m} \times$ $\mathrm{y}: 212.2 \mu \mathrm{m} \times \mathrm{z}: 25.0 \mu \mathrm{m})$ in XXWT and $X Y^{\text {sry-}}{ }^{-}$-female gonads (Student's $t$-test: ${ }^{* *} P<0.01$ for comparisons of the germ cell numbers made at E13.5, P1, and P8). (D) Representative images of immunostained germ cells at different developmental stages (E13.5, P1, and P8) in XXWT and XYsry--female gonads. PGCs and oocytes were stained with an antibody against a germ cell-specific marker (DDX4) and counterstained with DAPI (blue). Scale bar, $20 \mu \mathrm{m}$; magnification, $\times 40$. (E) Representative haematoxylin and eosin-stained sections of ovaries of 3- and 6-week-old XXWT and $X Y^{\text {sry- }}{ }^{\text {-females }}$ are shown. Scale bar, $200 \mu \mathrm{m}$.

Fig. 2. Extensive transcriptional changes in $X X Y^{\text {sry-}}$-female germ cells.

(A) Unsupervised hierarchical cluster of all transcript profiles $(n=36,172)$ from 6 RNA-Seq datasets. The height of the vertical axis indicates the Euclidean distance (correlation distance) between objects. (B) Germline-specific gene expression patterns in each germ cell RNA-Seq dataset. Asterisks indicate statistically significant differences between $X X$ WT and $X Y^{\text {sry-}}$-females (moderated $t$-test: ${ }^{* *} P<0.01$ ). (C) Gonadal somatic cell (GSC) marker gene 
expression patterns (5 medullary cell markers and 5 cortical cell markers) in each GSC RNA-Seq dataset. Asterisks indicate statistically significant differences between WT and $X Y^{\text {sry-}}$-females (moderated $t$-test: ${ }^{* *} P<0.01$ ). (D) The left two panels illustrate scatter plots of log2 RPKM values of WT (X-axis) and $X Y^{\text {sry- }}{ }^{-}$germ cell RNA-Seq samples ( $Y$-axis) obtained at E13.5 and P1. The right two panels illustrate scatter plots of log2 RPKM values of WT (X-axis) and $\mathrm{XY}^{\text {sry-}}$-GSC RNA-Seq samples (Y-axis) obtained at E13.5 and P1. Red circles indicate genes found to be differentially expressed ( $\geq$ two fold difference in the expression level) according to the moderated $t$-test at $P<0.05$.

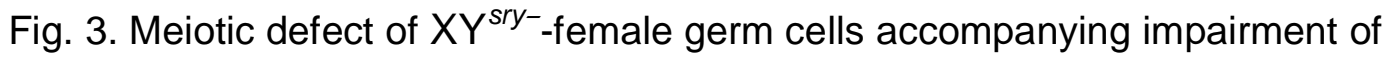
cell cycle progression and retrotransposable element activation.

(A) Expression patterns of Wnt signalling pathway components in each E13.5 PGC RNA-Seq dataset. Asterisks indicate statistically significant differences between RPKM values in E13.5 female, $X Y^{\text {sry-}}$-female and male PGCs (one-way ANOVA, ${ }^{* *} P<0.01$ ). (B) Representative images of immunostained E13.5 gonads. PGCs were stained with antibodies against a germ cell-specific marker (TRA98) and $\beta$-catenin, and counterstained with DAPI (blue). Arrowheads show $X^{\text {sry-}}{ }^{-}$-female $P G C$ s expressing stabilised $\beta$-catenin in the nucleus. Scale bar, $20 \mu \mathrm{m}$; magnification, $\times 40$. (C) Expression patterns of meiosis-related genes in each E13.5 PGC RNA-Seq dataset. Asterisks indicate statistically significant differences between RPKM values in E13.5 female,

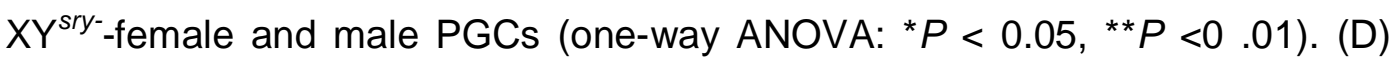
Representative images of immunostained E14.5 gonads. PGCs were stained with anti-TRA98 and anti-Ki67 (cell proliferation marker, red) antibodies, and counterstained with DAPI. Scale bar, $20 \mu \mathrm{m}$; magnification, $\times 40$. (E) Expression of representative retrotransposable elements in E13.5 and P1 germ cells. Data are represented by a heat map of the relative expression levels of each retrotransposable element. (F) Expression of Suv39h1 and Atrx in each germ cell RNA-Seq dataset. Asterisks indicate statistically significant differences 
between RPKM values in E13.5 female, $X Y^{\text {sry-}}$-female and male PGCs (one-way ANOVA, ${ }^{*} P<0.01$ ). (G) Representative images of immunostained

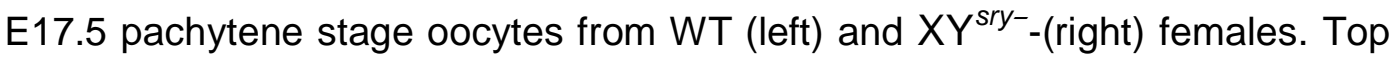
panel: oocytes were stained with anti-SCP3 (red) and anti-yH2AX (green) antibodies, and counterstained with DAPI. Dashed circle indicates yH2AX-positive region. No yH2AX signal was detected in XX WT oocytes (0/25; $0 \%$, whereas all $X Y^{\text {sry-}}$-oocytes were positive for $\mathrm{YH} 2 \mathrm{AX}(25 / 25 ; 100 \%)$. Bottom panel: Oocytes were stained with anti-SCP3 (red) and anti-SCP1 (green) antibodies and counterstained with DAPI. Dashed rectangles indicate the asynaptic regions. In XX WT oocytes, all homologous chromosomes have synapsed completely along their length at the pachytene stage. However, approximately a quarter of $X Y^{\text {sry-}}$-oocytes exhibited extensive chromosomal asynapsis $(6 / 25 ; 24.0 \%)$.

Fig. 4. Retardation of oocyte cyst breakdown and elimination of primordial follicles in neonatal $X Y^{\text {sry-}}{ }^{\text {-ovaries. }}$

(A) Representative Masson Trichrome (MT) sections of ovaries of P1 XX WT and $X Y^{\text {sry-}}$-females are shown. Bottom panels are magnified images of dashed rectangle depicted in upper panels. Arrows/dashed circles and arrowheads indicate oocyte cysts and primordial follicles, respectively. Scale bar, $100 \mu \mathrm{m}$. (B) TUNEL assay. Representative images of immunostained P1 ovaries in XX WT and $X Y^{\text {sry-}}{ }^{-}$-females. Ovaries were stained with antibody against a germ cell-specific marker (DDX4, red) and TUNEL (apoptotic cell marker, green). Dashed circles indicated by an allow indicate oocyte cysts and arrowheads indicate primordial follicles. Scale bar, $20 \mu \mathrm{m}$; magnification, $\times 40$. (C) Percentage contribution of oocyte in cysts and oocyte in follicles from P1 XX and $X Y^{\text {sry-}}$-ovaries. Asterisk indicates statistically significant differences between the groups in the rating of "oocyte in cysts" and "oocyte in follicles (Chi-squared test: ${ }^{* *} P<0.01, \mathrm{n}=3$ ). (D) Percentage of TUNEL positive oocytes in $\mathrm{P} 1 \mathrm{XX}$ and $X Y^{\text {sry-}}$-ovarian section. The percentage of apoptotic oocytes (TUNEL positive) 
was shown as the mean \pm standard error of the mean. Asterisk indicates a statistically significant difference (Student's $t$-test: ${ }^{* *} P<0.01, n=3$ ). (E) Expression patterns of folliclogenesis-related genes in each P1 oocyte RNA-Seq dataset. Asterisks indicate statistically significant differences between RPKM values in XX WT and $X Y^{\text {sry-}}$-oocytes (moderated $t$-test: ${ }^{* *} P<0.01$ ). (F) Representative MT sections of ovaries of P8 XX WT and $X Y^{\text {sry--females are }}$ shown. Bottom panels are magnified images of the dashed rectangle depicted in the upper panels. Arrows and arrowheads indicate primordial follicles and primary follicles, respectively. Scale bar, $100 \mu \mathrm{m}$. (G) Number of respective oocytes per 1 ovarian section at P8. Asterisks indicate a statistically significant difference between $\mathrm{XX}$ WT and $X \mathrm{Y}^{\text {sry-}}{ }^{-}$-oocytes (Student's $t$-test: ${ }^{* *} P<0.01, n=$ 3).

Fig. 5. Expression of Y-linked genes in E13.5 PGCs and P1 oocytes of $X Y^{\text {sry-}}$-females.

(A) Schematic diagram of the mouse $Y$ chromosome showing the male-specific region of the $Y$ short arm (MSYp; represented to scale in the magnified view), male specific region of the $Y$ long arm (MSYq), and pseudoautosomal region (PAR). The MSYp region has seven single copy genes, two duplicated genes, and one multi-copy gene. The MSYq region carries several multi-copy genes. The PAR is the chromosomal region, where sex $(X-Y)$ chromosome pairing occurs. (B) Expression patterns of $Y$-linked genes from E13.5 and P1 germ cell RNA-Seq datasets.

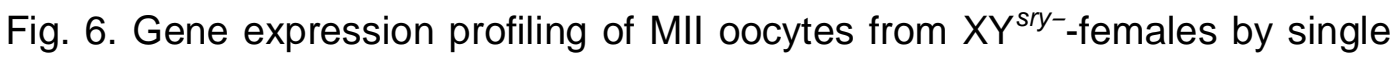
cell transcriptional analysis.

(A) Bi-dimensional PCA of gene expression profiles of XX WT $(n=30)$ and $X Y^{\text {sry- }}(\mathrm{n}=27)$ single oocyte. The bulk oocytes (20 oocytes, $\left.n=2\right)$ data were 
shown in square. The first principal component (PC1) captures $94.4 \%$ of the gene expression variability and the second principal component (PC2) captures $1.3 \%$ oocytes, respectively. (B) Boxplots of log2 RPKM values for 12 representative maternal effect genes in $\mathrm{XX} W \mathrm{WT}$ (pink) and $\mathrm{XY}^{\text {sry-}}{ }^{\text {-oocytes }}$ (purple). No significant difference was observed for each gene expression. (C) Heatmap shows relative expression of the 902 commonly up-regulated and 874 down-regulated genes in each $X Y^{\text {sry-}}$-MII oocyte identified by k-means clustering. Representative GO terms and the enrichment $P$ values are shown at right. (D) Heatmap shows relative expression of the 20 L1 subfamilies, 3 ERV2 elements and 3 satellite DNAs identified using k-means clustering methods, in $X \mathrm{Y}^{\text {sry-}}{ }^{-}$-MII oocytes. Blue dashed rectangle indicates a cluster, consisting of

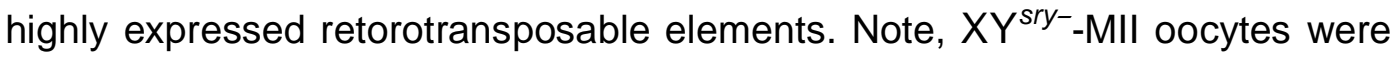
highly assigned to this cluster (18/19 (94.7\%), Fisher's exact test; $P<0.01)$. 


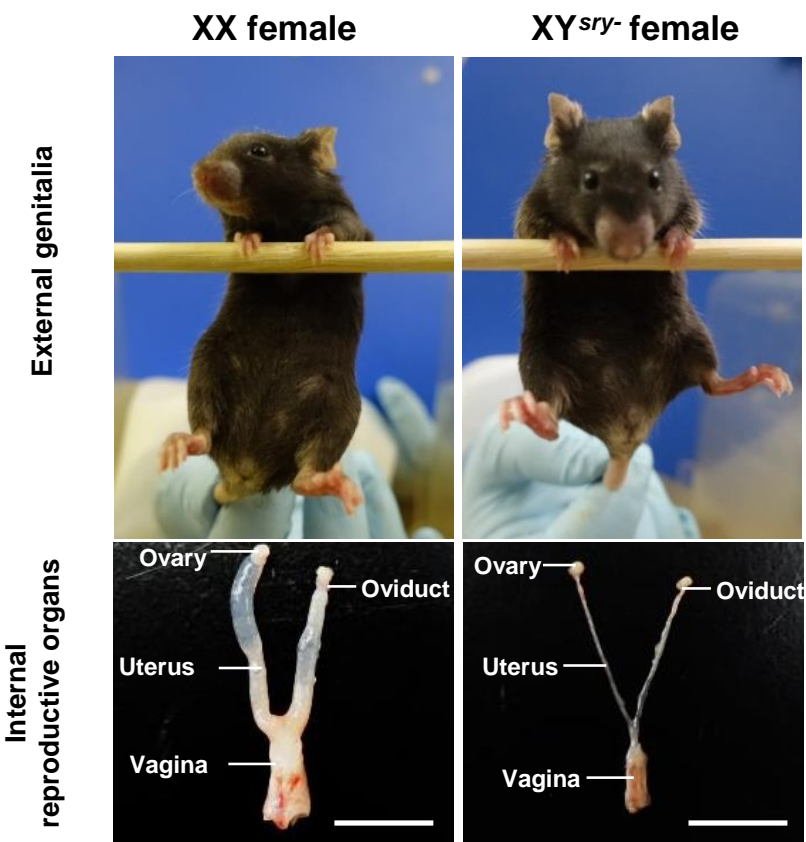

C
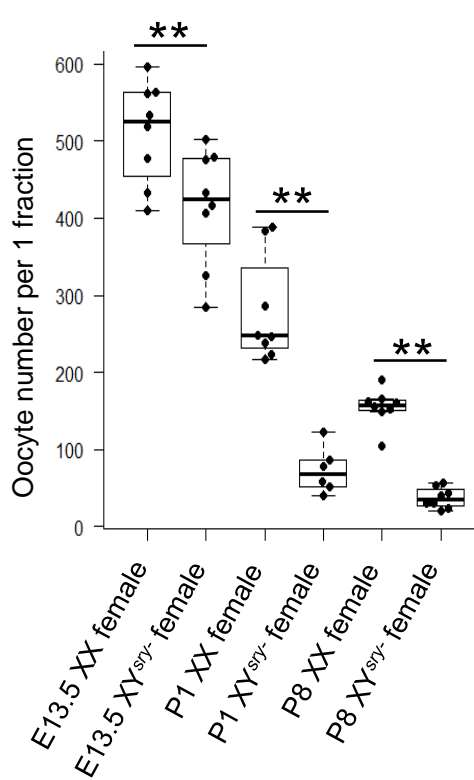

D

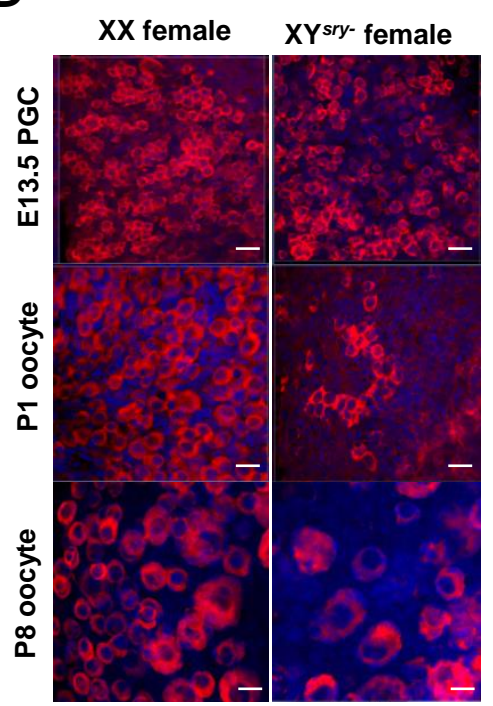

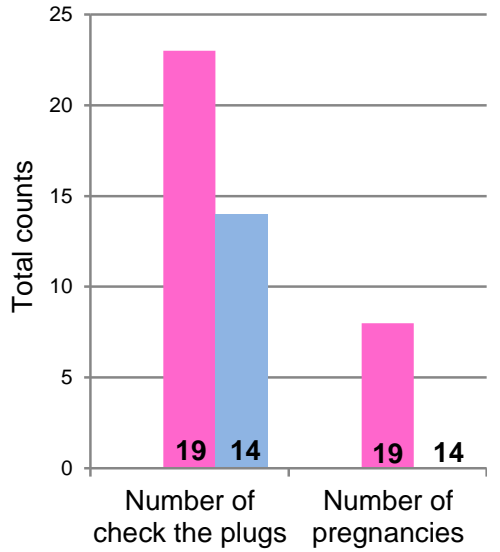

$X X$ female $X Y^{\text {sry- female }}$

E
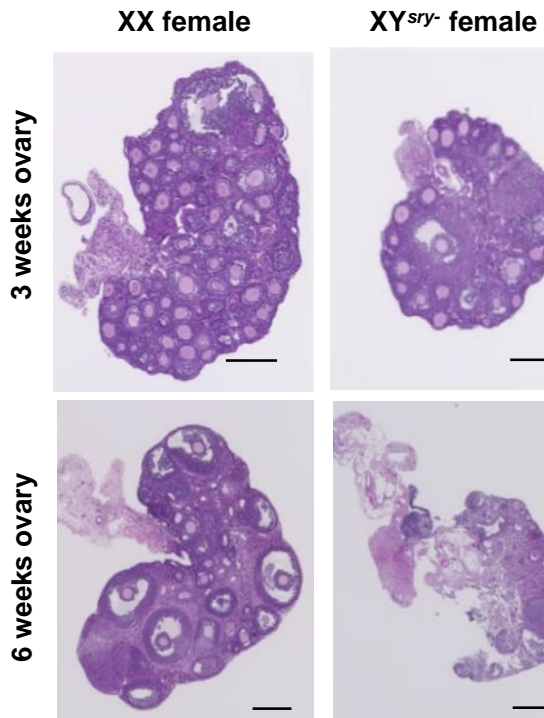

XYsry- female
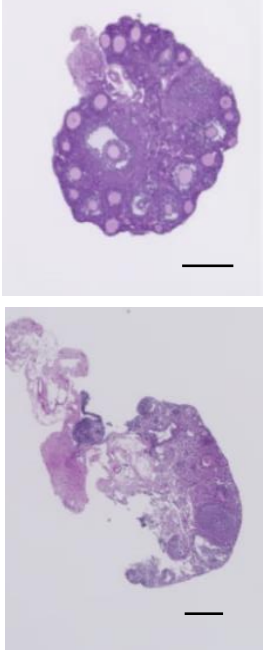

Fig. 1. 


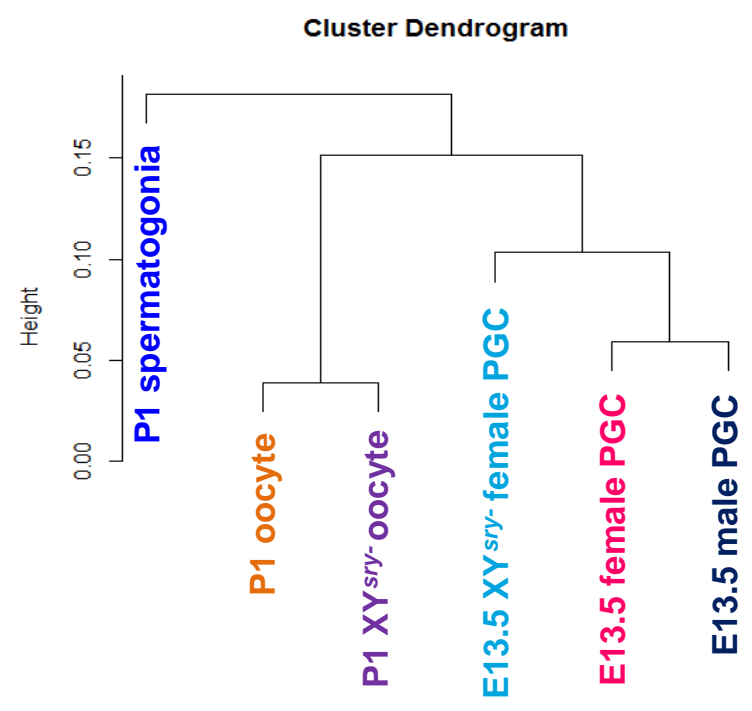

D

Gene expression profile at E13.5 PGC

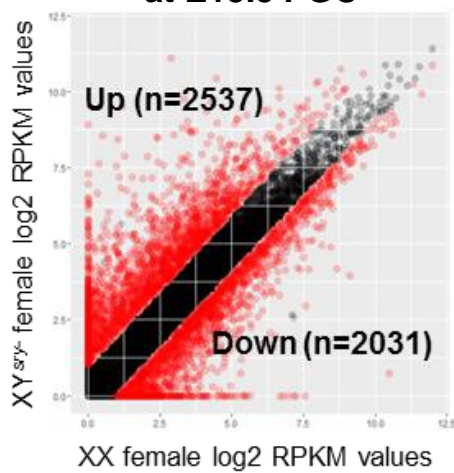

Fig. 2.
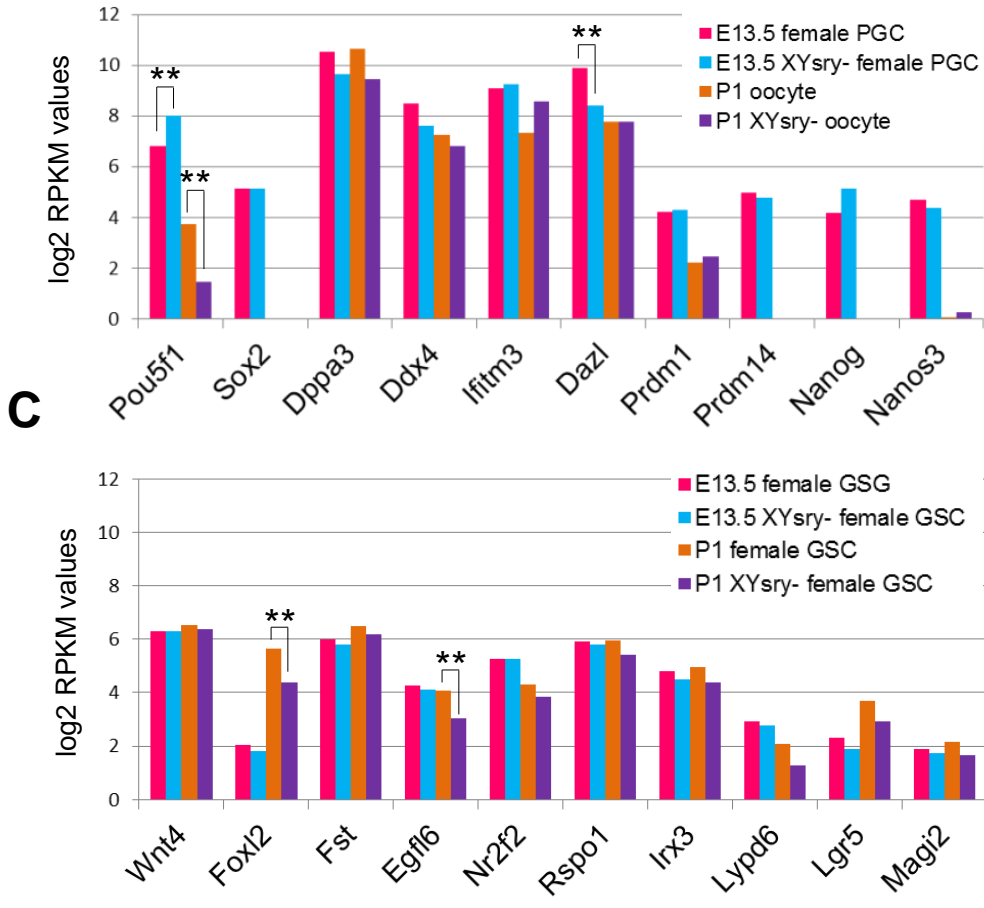

Medullary region

Cortical region

Gene expression profile Gene expression profile at P1 oocyte

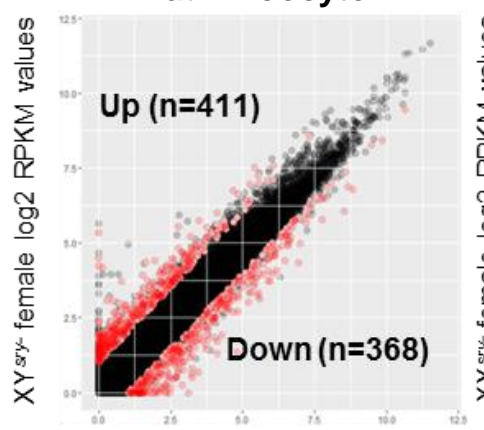

$\mathrm{XX}$ female log2 RPKM values at E13.5 GSC

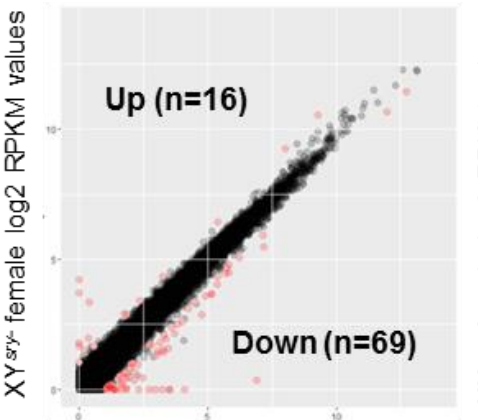

XX female log2 RPKM values
Gene expression profile at P1 GSC

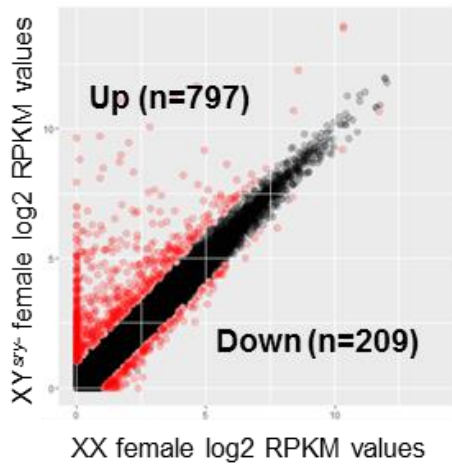


A

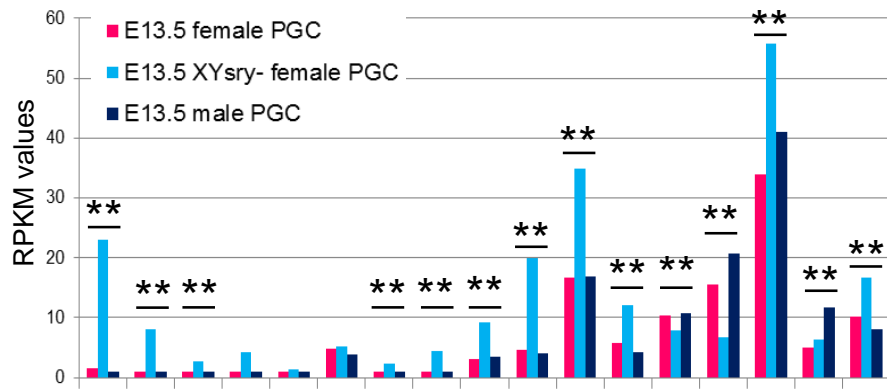

$\beta$-catenin

$X X$ female $\quad X Y$ sry- female $\quad X Y$ male

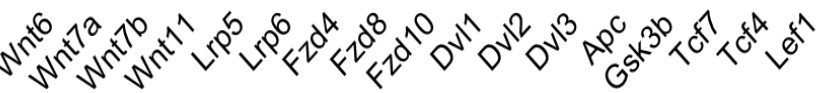

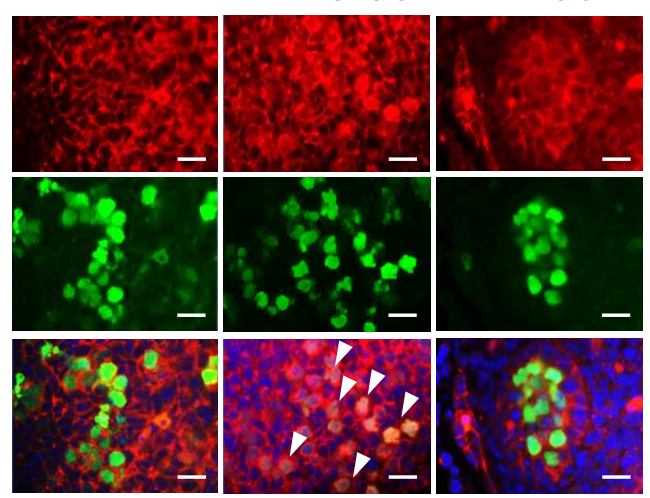

C

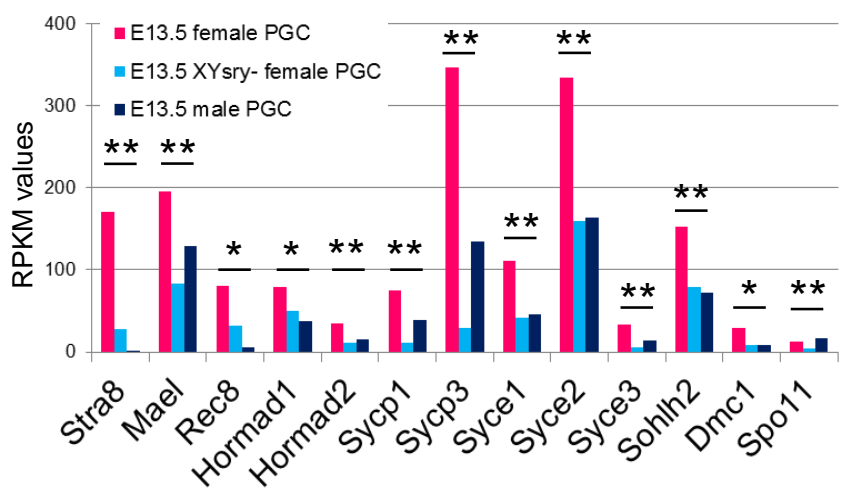

E

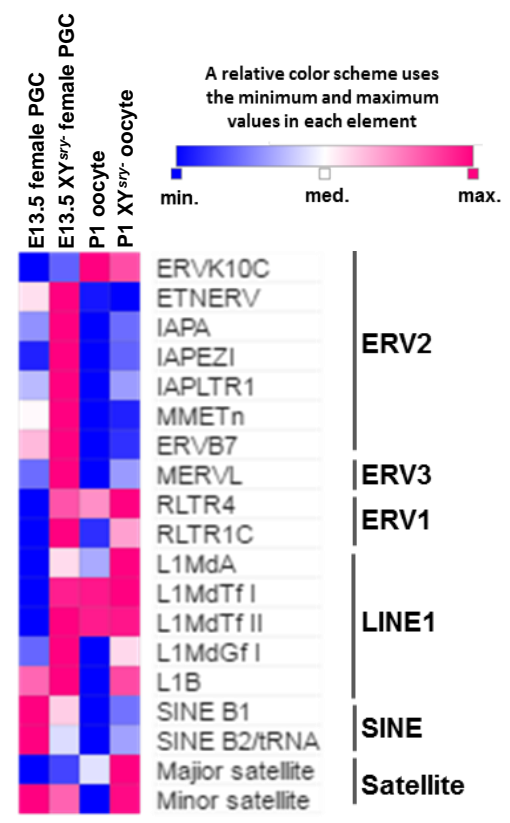

MERGE
D

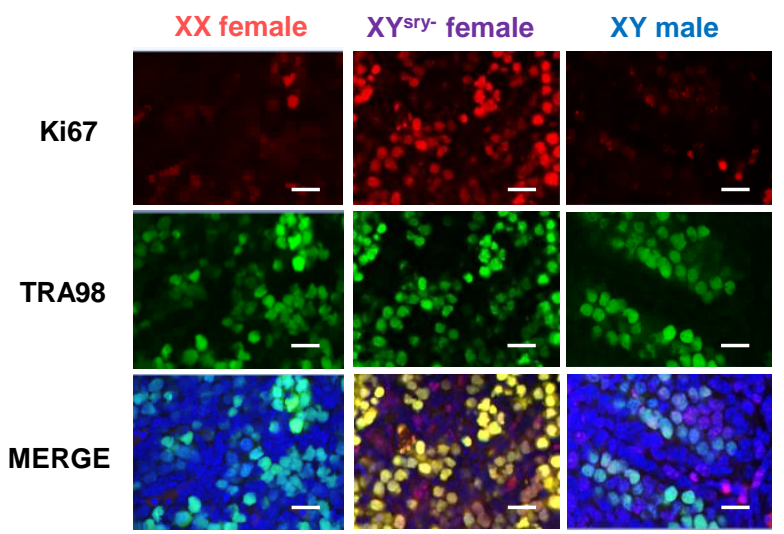

$F$

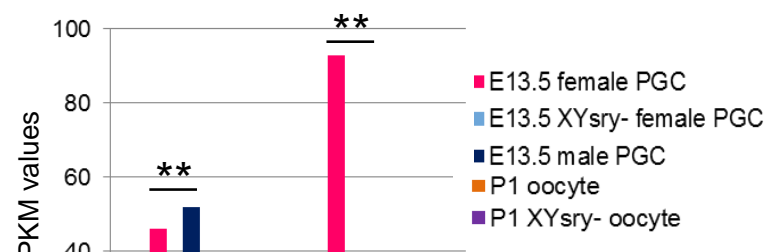

SCP3

YH2AX

DAPI

SCP3

SCP1

DAPI

G

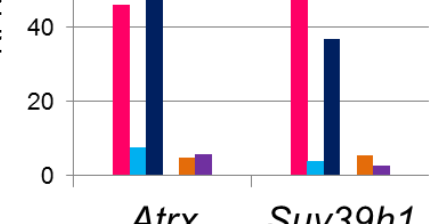

Atrx

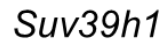

\section{XX female

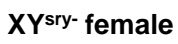

TRA98
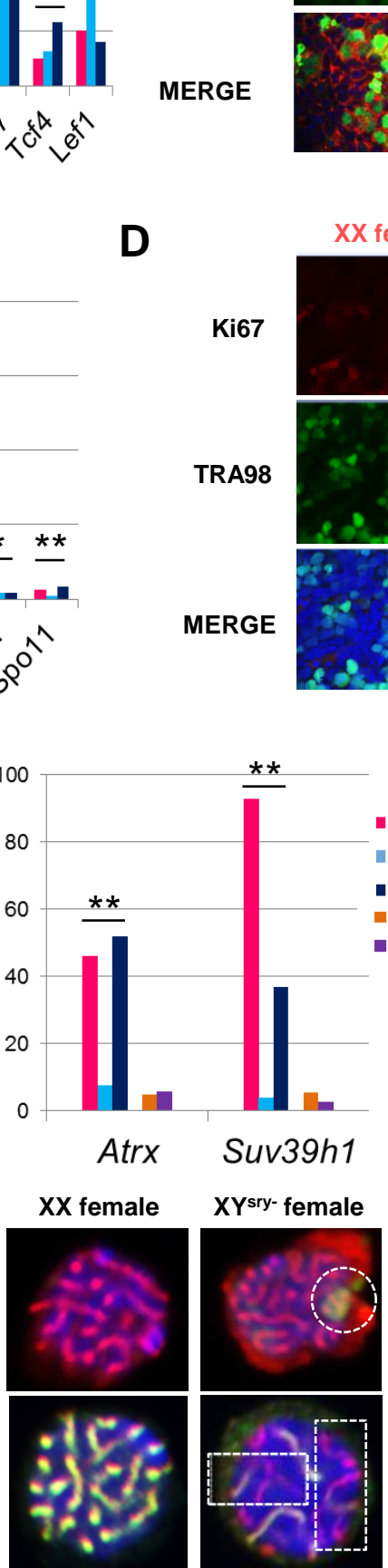

Fig. 3. 
A

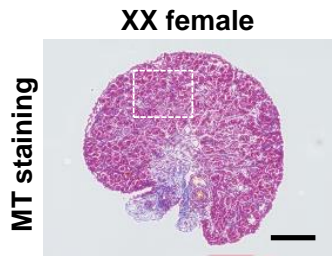

B
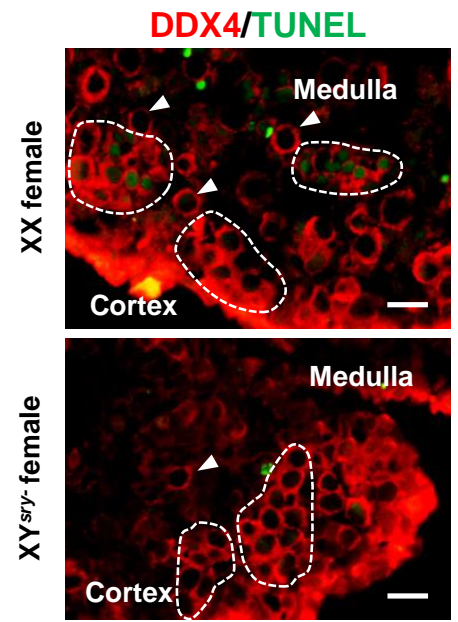

F
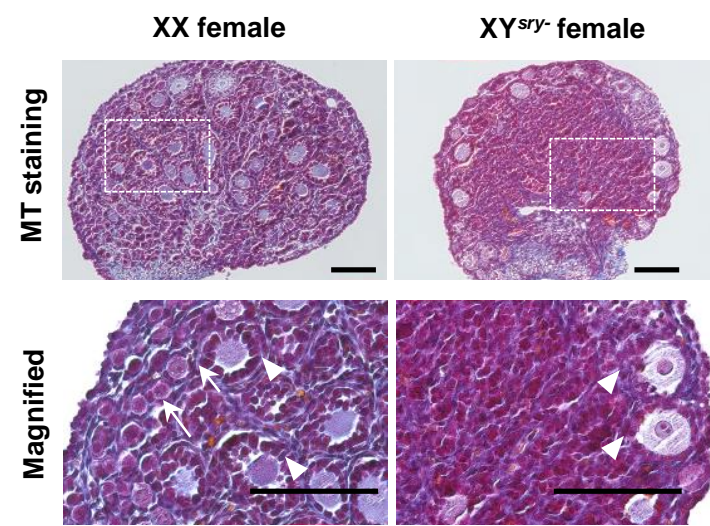

C XYsry- female

E

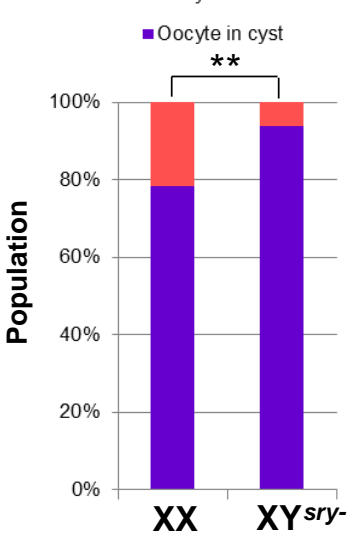

D

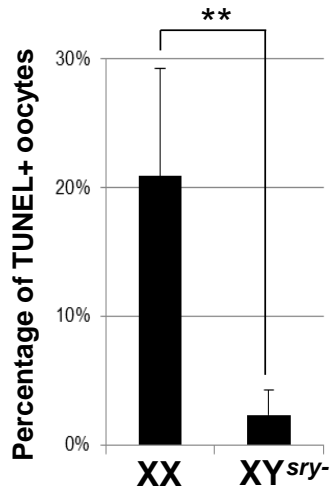

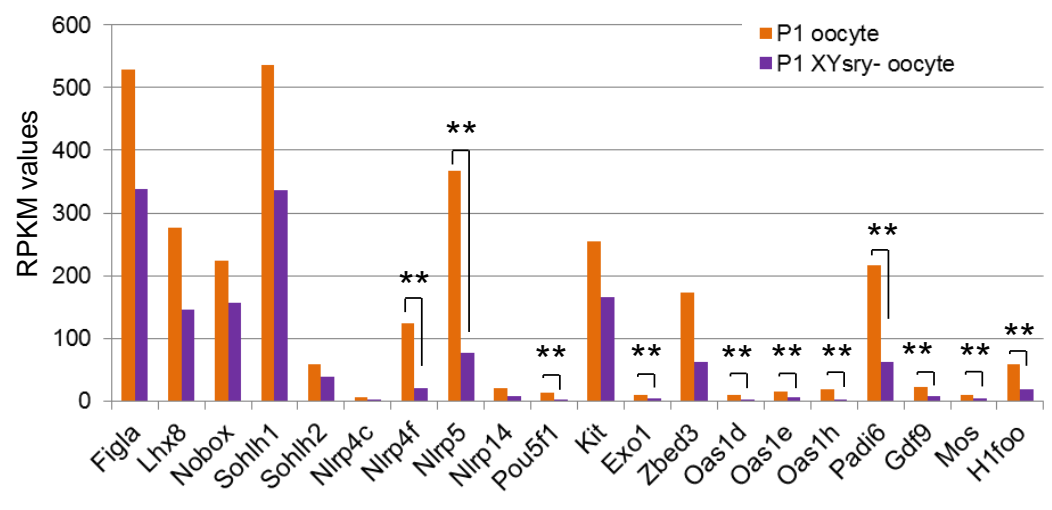

G

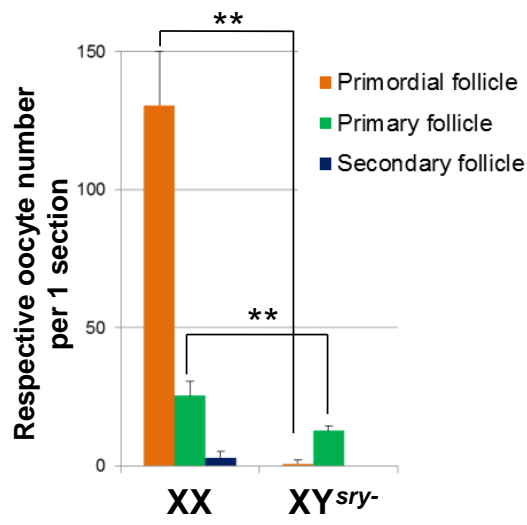

Fig. 4. 
A

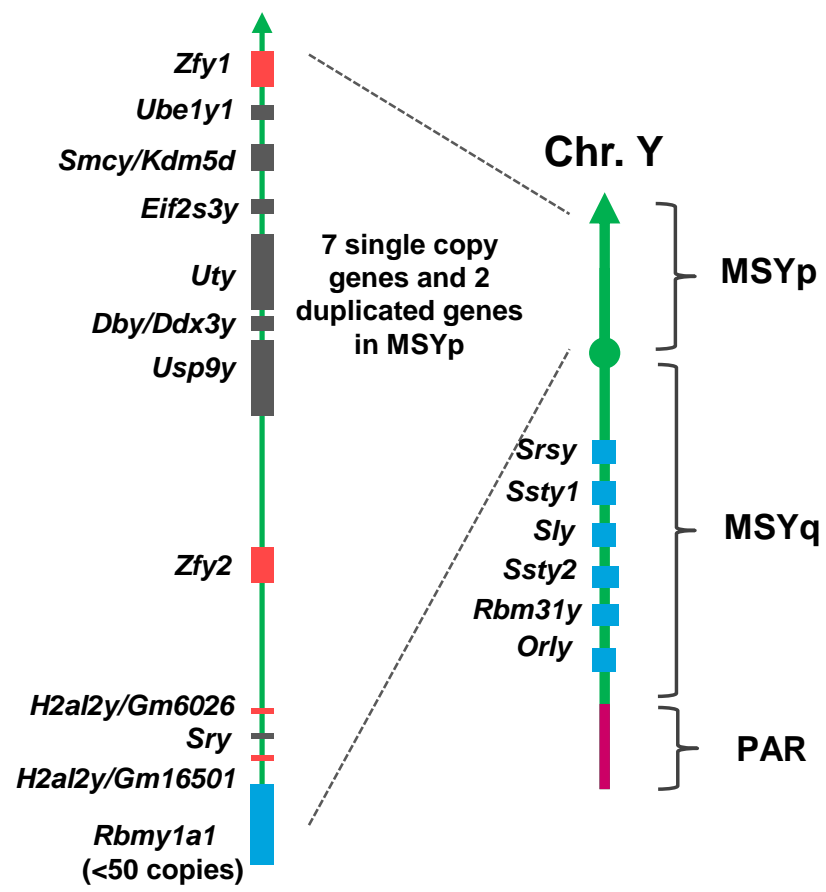

B

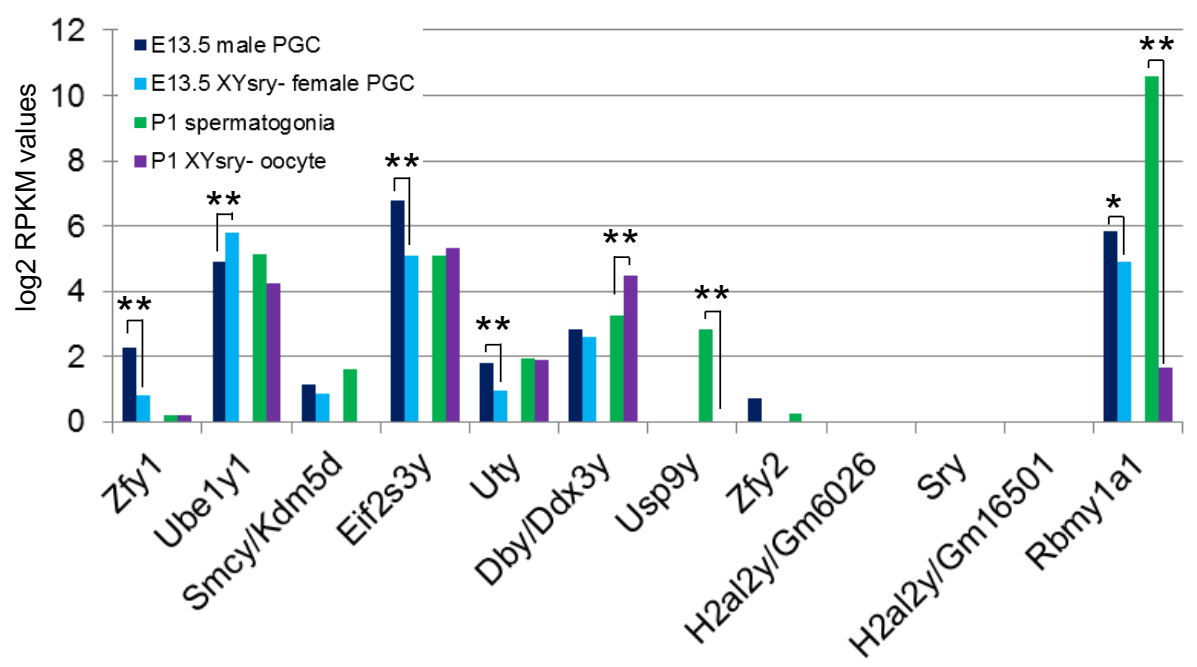

Fig. 5. 
A

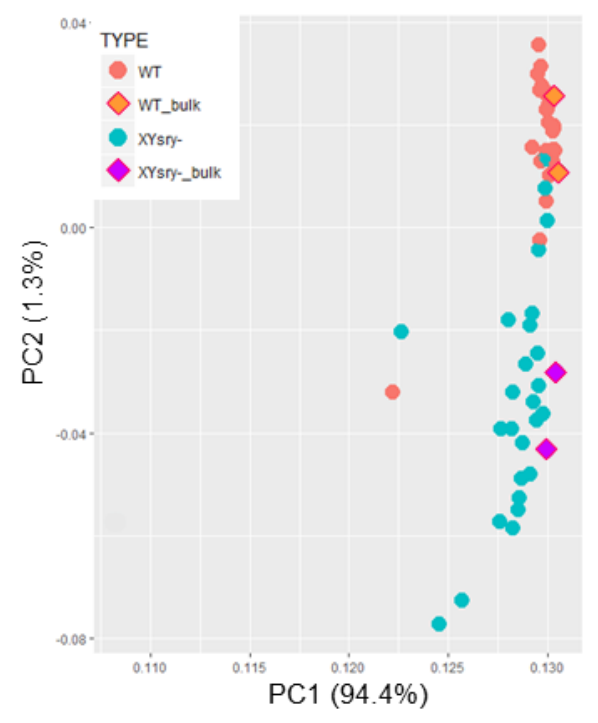

B

Representative maternal effect genes (related to competency for early embryo development)
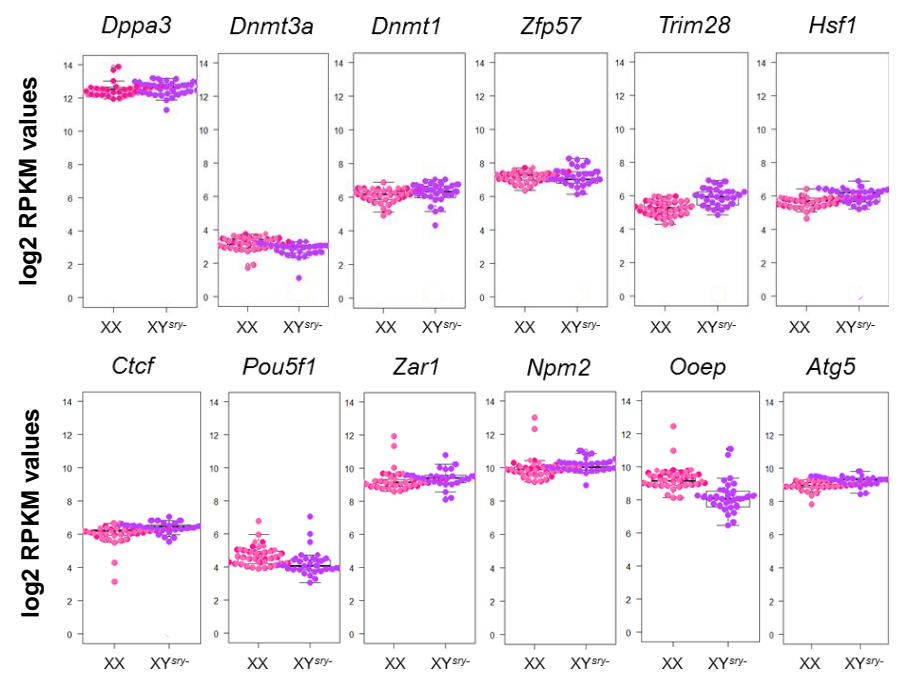

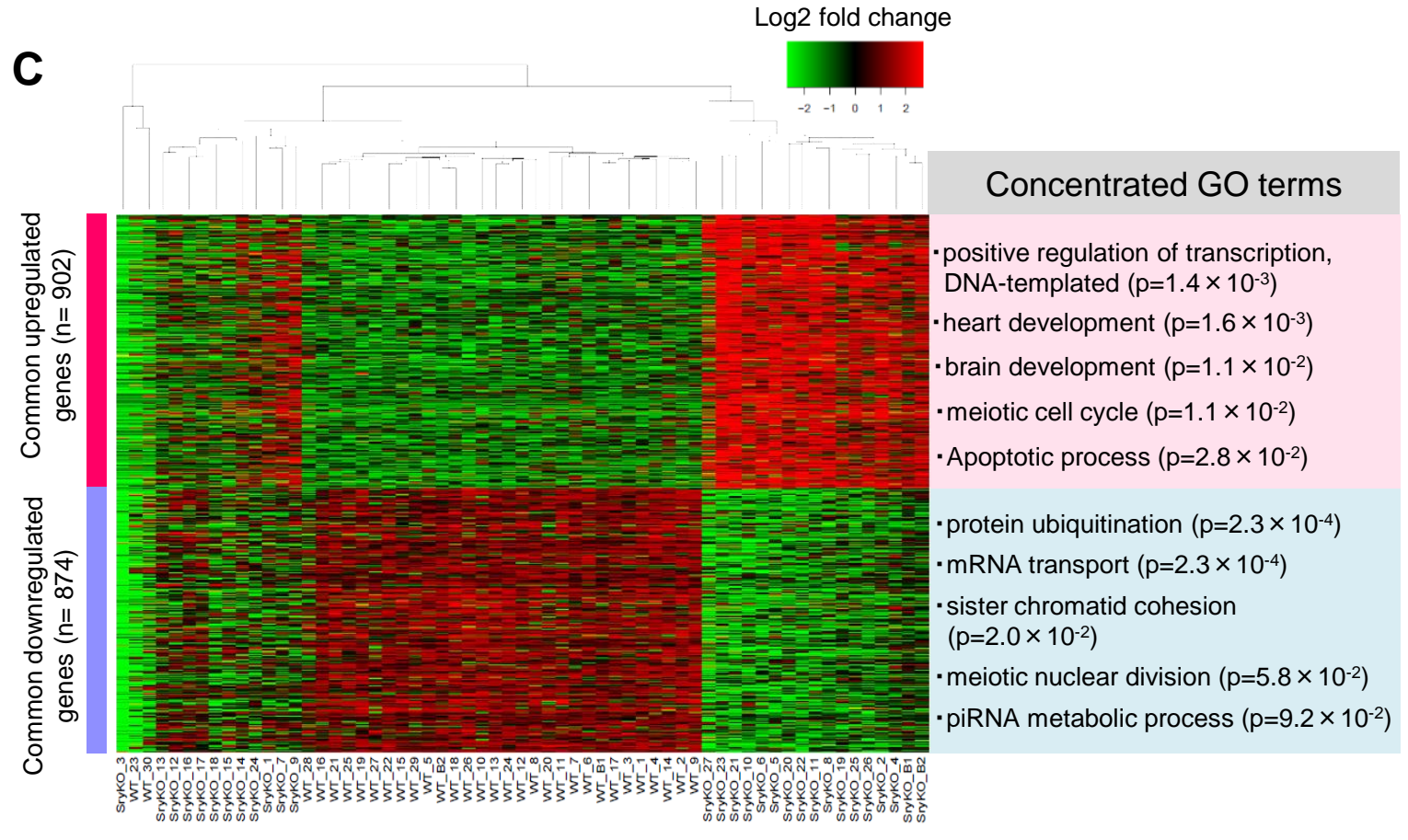

D

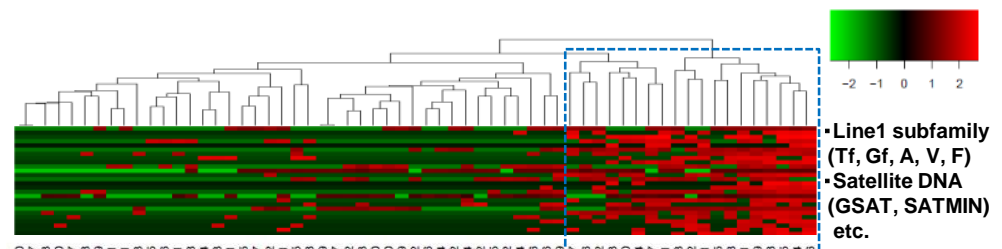

D.

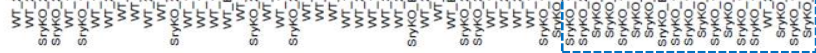

